\title{
Interferometric full-waveform inversion
}

\author{
Mrinal Sinha ${ }^{1}$ and Gerard T. Schuster ${ }^{1}$
}

\begin{abstract}
Velocity errors in the shallow part of the velocity model can lead to erroneous estimates of the full-waveform inversion (FWI) tomogram. If the location and topography of a reflector are known, then such a reflector can be used as a reference reflector to update the underlying velocity model. Reflections corresponding to this reference reflector are windowed in the data space. Windowed reference reflections are then crosscorrelated with reflections from deeper interfaces, which leads to partial cancellation of static errors caused by the overburden above the reference interface. Interferometric FWI (IFWI) is then used to invert the tomogram in the target region, by minimizing the normalized waveform misfit between the observed and predicted crosscorrelograms. Results with synthetic and field data with static errors above the reference interface indicate that an accurate tomogram can be inverted in areas lying within several wavelengths of the reference interface. IFWI can also be applied to synthetic time-lapse data to mitigate the nonrepeatability errors caused by time-varying overburden variations. The synthetic- and field-data examples demonstrate that IFWI can provide accurate tomograms when the near surface is ridden with velocity errors.
\end{abstract}

\section{INTRODUCTION}

Multiscale full-waveform inversion (MSFWI) can be used to resolve the low- and high-wavenumber characteristics of the elastic properties of the subsurface (Bunks et al., 1995; Boonyasiriwat et al., 2010; Fichtner et al., 2013). This makes FWI an ideal choice for inverting the fine-scaled characteristics of the subsurface medium. The success of FWI in resolving these features depends upon the availability of certain factors: low-frequencies and long offsets in the seismic data and a sufficiently accurate starting model.
We need to rely on reflections to invert for the velocity model in the deeper areas of the subsurface. Methods such as first-arrival tomography and early-arrival waveform inversion can be used to estimate the starting model for FWI of reflection data, but the kinematic information provided by these methods is mostly confined to the shallow parts. To recover a more accurate starting velocity model in the deeper areas, we need to use reflection-based inversion methods (Murphy and Gray, 1999; Lambaré, 2008; Guo and Schuster, 2017). These methods mainly provide low-wavenumber updates to the tomogram and might be unable to resolve small-scale velocity anomalies in the shallow subsurface because they mostly rely on long-wavelength updates. These unresolved small-scale anomalies can result in erroneous imaging of the deeper reflections. If we try to use a tomogram inverted from one of the aforementioned methods as our starting model for reflection FWI, chances are that the inversion would be hampered due to cycle skipping.

Zhou et al. (2006) introduce the concept of interferometric migration (IM) to mitigate the defocusing due to the statics in the data. In this method, they shift the data by the traveltimes of the picked reference reflections. This time shift can also be automatically computed by crosscorrelating the trace windowed around the reference reflection with the original trace. This procedure is carried out for all the traces using 1D time-domain crosscorrelation to theoretically cancel out the phase associated with the common raypaths above the reference interface for small source-receiver offsets. It approximately redatums the data to the reference interface without using a known velocity model. One problem, however, is that the correlated traces can lead to artifacts in the migration image and the reference reflections must be carefully windowed. To overcome this problem, the interferometric least-squares migration (ILSM) method was proposed by Sinha and Schuster (2016), where the migration artifacts are significantly reduced and improved image resolution is achieved by iterative least-squares migration. We now extend the ILSM method to waveform inversion for mitigating near-surface problems and inversion of time-lapse data. For time-lapse data, the reference reflector is selected and the correlated data are migrated at each iteration. Unlike ILSM, the background slowness is updated at each

\footnotetext{
Manuscript received by the Editor 17 January 2018; revised manuscript received 24 July 2018; published ahead of production 01 October 2018; published online 04 December 2018

${ }^{1}$ King Abdullah University of Science and Technology, Department of Earth Science and Engineering, Thuwal 23955 6900, Saudi Arabia. E-mail: mrinal .sinha@kaust.edu.sa; gerard.schuster@kaust.edu.sa.
}

(C) 2019 Society of Exploration Geophysicists. All rights reserved. 
iteration. The approximate redatuming property of the reference reflections makes it suitable for applications, such as target-oriented waveform inversion and estimation of time-lapse changes.

The main aim of 4D seismic surveys is to seismically detect the location of fluid movement in reservoirs caused by production activities. One of the problems with 4D seismic surveys is the timevarying environmental conditions, which can render the experiment insufficiently repeatable. The water-layer velocity during a marine experiment varies with time because of the time-varying environmental conditions, such as temperature, salinity, ocean currents, and depth (MacKay et al., 2003). For deepwater reservoirs, these variations are even more prominent because the water column is very thick. These deviations lead to uncertainty in the estimation of the water-layer velocity, which can lead to image distortion similar to that caused by static errors in land data. In addition, tidal variations will introduce statics shifts into marine data. Recently, many applications of 4D FWI for recovering temporal model variations have appeared in the literature (Routh et al., 2012; Queisser and Singh, 2013; Raknes and Arntsen, 2014; Kamei and Lumley, 2017), but FWI is sensitive to nonrepeatability errors (Asnaashari et al., 2012). Maharramov et al. (2016) simultaneously invert the baseline and monitor data sets, while also imposing a model-regularization constraint to counter nonrepeatability problems. We aim to invert for the time-lapse changes by naturally redatuming the data to a reference interface below the overburden and thereby negating the deteriorative effects of the nonrepeatability errors. We denote this as natural redatuming because the recorded reference reflections are used for the redatuming operation.

The paper is organized into five sections. The introduction is followed by the theory of interferometric FWI (IFWI). The formulas for IFWI are similar to those for ILSM (Sinha and Schuster, 2016), except the background slowness model is updated at each iteration. Numerical results are then presented in the next section, followed by a discussion and conclusions.

\section{THEORY OF IFWI}

Let the predicted trace in the frequency domain be denoted by $D(\mathbf{g} \mid \mathbf{s})$ for a source at $\mathbf{s}$ and geophone at $\mathbf{g}$. Let $D(\mathbf{g} \mid \mathbf{s})_{\text {ref }}$ denote the predicted trace that is windowed around a reference reflection event, as illustrated in Figure 1. To estimate the predicted crosscorrelo- gram $\Phi(\mathbf{g} \mid \mathbf{s})$, the windowed reference reflections in the predicted data are temporally crosscorrelated with the predicted data. In the frequency domain, crosscorrelation is equivalent to the spectral product

$$
\Phi(\mathbf{g} \mid \mathbf{s})=D(\mathbf{g} \mid \mathbf{s}) D^{*}(\mathbf{g} \mid \mathbf{s})_{\text {ref }},
$$

which removes the two-way propagation time from the surface to the reflector for near-offset traces. For example, denote the two-way propagation time to the reference interface as $\tau_{\text {cal }}^{\text {ref }}$, so that $D(\mathbf{g} \mid \mathbf{s})_{\text {ref }}=e^{i \omega\left(\tau_{\text {cal }}^{\text {ref }}\right)}$. If the reflection data from a deeper interface are given by $D(\mathbf{g} \mid \mathbf{s})=e^{i \omega\left(\tau_{\text {cal }}^{x_{0}}\right)}$, then $D(\mathbf{g} \mid \mathbf{s}) D(\mathbf{g} \mid \mathbf{s})_{\mathrm{ref}}^{*}=$ $e^{i \omega\left(\tau_{\text {cal }}^{x_{0}}-\tau_{\text {cal }}^{\text {ref }}\right)}$. Thus, the deep-reflection data have been naturally redatumed to the reference reflector without knowing the velocity model. Similarly, the observed crosscorrelogram $\Phi_{\text {obs }}(\mathbf{g} \mid \mathbf{s})$ can be obtained by the crosscorrelation of recorded traces with the observed reference reflection traces. However, the implicit assumption is that the reflection rays for the reference reflection coincide with a portion of the rays associated with the deep reflection. The credibility of this assumption depends on the combination of two factors, namely, the depth of the imaged reflectors relative to the reference reflector and the source-receiver offset. This assumption becomes increasingly violated for longer-offset data and deeper reflections located more than 3-4 wavelengths away from the reference interface.

The goal is to find the slowness (velocity) model, which minimizes the $L_{2}$-norm of the difference between the normalized observed and predicted crosscorrelograms in the frequency domain

$$
\epsilon=\frac{1}{2} \sum_{\omega} \sum_{s} \sum_{g}\left\|\hat{\Phi}(\mathbf{g} \mid \mathbf{s})-\hat{\Phi}_{\mathrm{obs}}(\mathbf{g} \mid \mathbf{s})\right\|^{2},
$$

where $\quad \hat{\Phi}(\mathbf{g} \mid \mathbf{s})=\Phi(\mathbf{g} \mid \mathbf{s}) /\|\Phi(\mathbf{g} \mid \mathbf{s})\|, \quad \hat{\Phi}_{\text {obs }}(\mathbf{g} \mid \mathbf{s})=\Phi_{\text {obs }}(\mathbf{g} \mid \mathbf{s}) / \|$ $\Phi_{\text {obs }}(\mathbf{g} \mid \mathbf{s}) \|$, and the angular-frequency notation is silent. The gradient of equation 2 with respect to the perturbation in slowness $s(\mathbf{x})^{\text {slow }}$ is

$$
\begin{aligned}
\frac{\partial \epsilon}{\partial s(\mathbf{x})^{\text {slow }}}= & \sum_{\omega} \sum_{s} \sum_{g} \Re\left[\frac{\partial \Phi(\mathbf{g} \mid \mathbf{s})}{\partial s(\mathbf{x})^{\text {slow }}} \frac{1}{\|\Phi(\mathbf{g} \mid \mathbf{s})\|}[w(\mathbf{g} \mid \mathbf{s}) \hat{\Phi}(\mathbf{g} \mid \mathbf{s})\right. \\
& \left.\left.-\hat{\Phi}_{\text {obs }}(\mathbf{g} \mid \mathbf{s})\right]\right]
\end{aligned}
$$

Figure 1. Model settings for (a) observed and (b) predicted data. The observed data are windowed around the reference reflection event to extract the observed reference reflection. a)

Observed data

b)

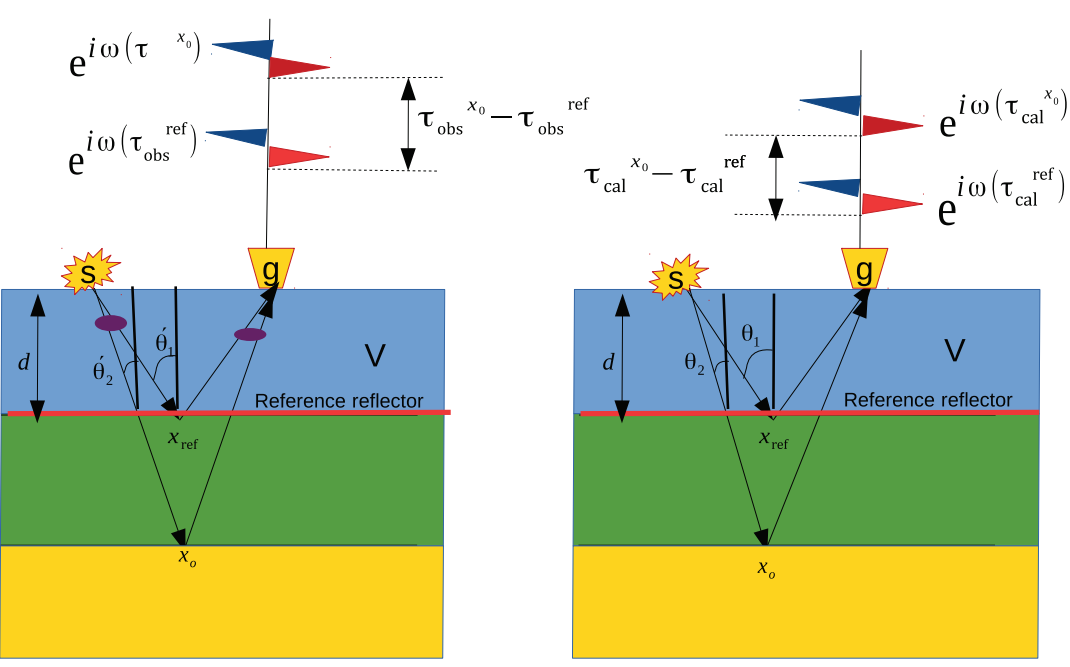


where $w(\mathbf{g} \mid \mathbf{s})$ is the weight given by $w(\mathbf{g} \mid \mathbf{s})=\hat{\Phi}(\mathbf{g} \mid \mathbf{s})^{*} \hat{\Phi}_{\text {obs }}(\mathbf{g} \mid \mathbf{s})$, which emphasizes the phase mismatch between the observed and the predicted crosscorrelograms and reduces the reliance on the absolute amplitudes. If the two crosscorrelograms are perfectly aligned with each other, then $w(\mathbf{g} \mid \mathbf{s})=1$. The summand in equation 3 is a Hermitian function, which implies that its $\Re$ and $\mathfrak{I}$ parts are even and odd functions of $\omega$, respectively. Therefore, summation over frequencies will result in cancellation of the $\mathfrak{s}$ part, so that the $\Re$ notation will be dropped in the forthcoming equations. Substituting the expression for predicted crosscorrelograms in equation 1 into equation 3 gives

$$
\begin{gathered}
\frac{\partial \epsilon}{\partial s(\mathbf{x})^{\text {slow }}}=-\sum_{\omega} \sum_{\mathbf{s}} \sum_{\mathbf{g}} \overbrace{\frac{\partial D^{*}(\mathbf{g} \mid \mathbf{s})}{\partial s(\mathbf{x})^{\text {slow }}}}^{\text {Migration kernel }} \\
\times \overbrace{\frac{1}{\|\Phi(\mathbf{g} \mid \mathbf{s})\|} D(\mathbf{g} \mid \mathbf{s})_{\text {ref }}\left[w(\mathbf{g} \mid \mathbf{s}) \hat{\Phi}(\mathbf{g} \mid \mathbf{s})-\hat{\Phi}_{\text {obs }}(\mathbf{g} \mid \mathbf{s})\right]}^{\text {Interferometric residual }=\Delta \Phi},
\end{gathered}
$$

where the Fréchet derivative $\partial D(\mathbf{g} \mid \mathbf{s}) / \partial s(\mathbf{x})^{\text {slow }}$ for an acoustic medium is given by Schuster (2017) as

$$
\frac{\partial D(\mathbf{g} \mid \mathbf{s})}{\partial s(\mathbf{x})^{\text {slow }}}=2 \omega^{2} s(\mathbf{x})^{\text {slow }} G(\mathbf{g} \mid \mathbf{x}) G(\mathbf{x} \mid \mathbf{s}) W(\omega),
$$

and $W(\omega)$ is the spectrum of the source wavelet.

The Fréchet derivative corresponding to the term $\partial D(\mathbf{g} \mid \mathbf{s})_{\text {ref }} /$ $\partial s(\mathbf{x})^{\text {slow }}$, which results from the application of the chain rule to $\partial \Phi(\mathbf{g} \mid \mathbf{s}) / \partial s(\mathbf{x})^{\text {slow }}$, can be automatically set to zero by keeping the velocity model above the reference interface fixed. To avoid amplitude mismatches, the predicted magnitude spectrum of the crosscorrelation function can be replaced with that of the observed spectrum (Fu et al., 2018).

Substituting equation 5 into equation 4 and replacing the interferometric residual by $\Delta \Phi$, we get

$$
\begin{gathered}
\frac{\partial \epsilon}{\partial s(\mathbf{x})^{\text {slow }}}=-2 \sum_{\omega} \sum_{\mathbf{s}} \sum_{\mathbf{g}} \omega^{2} s(\mathbf{x})^{\text {slow }} W(\omega)^{*} G(\mathbf{x} \mid \mathbf{s})^{*} G(\mathbf{g} \mid \mathbf{x})^{*} \Delta \Phi(\mathbf{g} \mid \mathbf{s}), \\
=-2 \sum_{\omega} \sum_{\mathbf{s}} \sum_{\mathbf{g}} \omega^{2} s(\mathbf{x})^{\text {slow }} \overbrace{[W(\omega) G(\mathbf{x} \mid \mathbf{s})]^{*}}^{\text {Source wavefield }} \overbrace{\left[G(\mathbf{g} \mid \mathbf{x})^{*} \Delta \Phi(\mathbf{g} \mid \mathbf{s})\right]}^{\text {Backpropagated wavefield }} .
\end{gathered}
$$

Equation 6 implies that the interferometric gradient is formed by smearing the interferometric residual along the associated migration ellipses and rabbit ears. Rabbit ears (Huang and Schuster, 2014) are reflection wavepaths formed from sharp interfaces in the velocity model. Replacing the observed and predicted data components in equation 4 by their respective high-frequency Green's function representations, we get

$$
\begin{gathered}
\frac{\partial \epsilon}{\partial s(\mathbf{x})^{\text {slow }}}=\sum_{\omega} \sum_{\mathbf{s}} \sum_{\mathbf{g}}[w(\mathbf{g} \mid \mathbf{s}) D(\mathbf{g} \mid \mathbf{s}) \\
\left.-D_{\mathrm{obs}}(\mathbf{g} \mid \mathbf{s}) e^{i \omega \overbrace{\left(\tau_{\text {cal }}^{\text {ref }}-\tau_{\text {obs }}^{\text {ref }}\right)}^{\delta_{\text {ref }} \text { timing error }}}\right] \overbrace{e^{-i \omega\left(\tau_{\mathbf{s x}}+\tau_{\mathbf{x g}}\right)}}^{\text {migration kernel }},
\end{gathered}
$$

where $\tau_{\mathbf{s} \mathbf{x}}$ and $\tau_{\mathbf{g x}}$ signify the traveltimes from the source $\mathbf{x}$ and receiver $\mathbf{g}$ to a trial image point $\mathbf{x}$ in the subsurface. The detailed derivation of equation 7 is given in Sinha and Schuster (2016), and it implies that $\delta^{\text {ref }}$ acts as a correction term to account for the unre- solved overburden anomalies. This correction is comprised of two parts: First, the data are approximately redatumed to the reference interface using the windowed reference reflections in the data and then the calculated reference reflection events redatum the data back to the recording surface. The crosscorrelation with the observed reference reflection traces removes the distortions caused by velocity and source-receiver positioning errors and the convolution with the predicted reference data adjust the observed data to conform with the available velocity model and acquisition geometry. The sensitivity of IFWI to source-receiver offset, relative depth of target events, and the reference reflector depth is discussed in Appendix A.

\section{Workflow}

This section describes the workflow for IFWI:

- Select a reference reflector in the velocity model, and window the corresponding reference reflections in the observed data as shown by the yellow lines in Figure $2 \mathrm{a}$ and $2 \mathrm{~b}$, respectively. The predicted reference reflections are computed by Born modeling (Schuster, 2017), which uses the initial-velocity model and the chosen reference reflector as the reflectivity model. If the overburden velocity model has significant errors, the reflectors will be distorted from their actual shapes. In practice, the distorted reflector can be windowed from the migration image to obtain an estimate of observed reference reflections. This avoids tedious picking, even though the picked reference in the observed data will provide more accuracy.

- Crosscorrelate the recorded traces with the windowedobserved reference reflection traces to get the observed crosscorrelograms. Similarly, compute the predicted crosscorrelograms to calculate the interferometric residual in equation 4 . The observed and predicted crosscorrelograms are shown in Figure $2 \mathrm{c}$ and 2d, respectively.

- Calculate the gradient $\mathbf{g}^{k}$ in equation 4 and update the search direction $\mathbf{d}^{k}$ using the conjugate-gradient (CG) method (Nocedal and Wright, 2006)

$$
\mathbf{d}^{k}=-\mathbf{g}^{k}+\beta \mathbf{d}^{k-1} .
$$

The benefits of choosing the normalized $L_{2}$-norm misfit for IFWI over the conventional $L_{2}$-norm misfit are depicted in Figure 2e and 2f. Crosscorrelation operations alter the source signature in the crosscorrelograms, which can lead to artifacts in the unnormalized IFWI gradient. However, the normalized IFWI misfit relies on the phase mismatch between the crosscorrelogram waveforms and is thus able to avoid such artifacts.

- Compute the step length $\alpha$ by a line-search method.

- Update the slowness model $s(\mathbf{x})^{\text {slowk }}$ by

$$
s(\mathbf{x})^{\text {slow } k}=s(\mathbf{x})^{\text {slow } k-1}+\alpha \mathbf{d}^{k} .
$$

- Calculate the new predicted crosscorrelogram using equation 1 .

\section{Different strategies for time-lapse estimation}

Estimating temporal changes in the slowness is usually performed by inverting the baseline and monitor data sets separately, 
and then the inverted monitor and baseline tomograms are subtracted from each other for estimating the temporal velocity variations in the reservoir. The problem with this approach is that we might converge to two different local minima after inversion, and a subtraction of two such models will lead to spurious property changes.

Another approach is to use the velocity model inverted from the baseline data as an initial model for inverting the data from the monitor survey. Then, the two models are subtracted from one another to evaluate the time-lapse velocity change. This assumes that the estimated baseline model is complete to the extent that when used as an initial model for inverting the monitor data, all the extra updates are exclusively caused by reservoir changes. But in practice, we always generate extra nonreservoir updates because there are a finite number of iterations used for inverting the baseline model.

Watanabe et al. (2005), Asnaashari et al. (2012), Yang et al. (2016), and Kamei and Lumley (2017) use double-difference FWI (DDFWI) to invert for time-lapse velocity changes and show that DDWI provides a more accurate estimate for the time-lapse changes compared with the previously discussed strategies. In this strategy, the estimated baseline model is used as the initial model, but instead of inverting the monitor data as a whole, we invert the time-lapse differences between the baseline and monitor data sets. This is done by modifying the misfit as shown below:

$$
\begin{gathered}
J(m)=\sum_{\omega} \sum_{s} \sum_{g} \frac{1}{2} \|\left(\hat{D}_{\text {mon }}(\mathbf{g} \mid \mathbf{s})-\hat{D}_{\text {base }}(\mathbf{g} \mid \mathbf{s})\right) \\
-\left(\hat{u}_{\text {mon }}(\mathbf{g} \mid \mathbf{s}, m)-\hat{u}_{\text {base }}\left(\mathbf{g} \mid \mathbf{s}, m_{0}\right)\right) \|^{2},
\end{gathered}
$$

where $\hat{D}_{\text {mon }}(\mathbf{g} \mid \mathbf{s})$ and $\hat{D}_{\text {base }}(\mathbf{g} \mid \mathbf{s})$ represent the normalized observed monitor and baseline data for a source at $\mathbf{s}$ and geophone at $\mathbf{g}$, respectively; $\hat{u}_{\text {mon }}(\mathbf{g} \mid \mathbf{s}, m)$ denotes the normalized predicted data generated with the model $m ; \hat{u}_{\text {base }}\left(\mathbf{g} \mid \mathbf{s}, m_{0}\right)$ is the normalized synthetic data generated using the baseline model $m_{0}$ estimated by FWI; and $m$ represents the velocity model for the monitor survey. Because the baseline model $m_{0}$ has already been inverted for, $u_{\text {base }}$ remains fixed during DDWI. Similarly, by replacing the observed and predicted data by their respective crosscorrelogram components in equation 10, we obtain the misfit for double-difference IFWI (DDIFWI), as shown below:
Figure 2. A reference reflector is chosen in (a) model space and its corresponding (b) reflection event is windowed (yellow) in observed data. (c) Observed and (d) predicted crosscorrelograms are evaluated to estimate the interferometric residual. (f) The normalized $L_{2}$-norm gradient provides a better conditioned gradient than the (e) conventional $L_{2}$-norm gradient for IFWI. a)

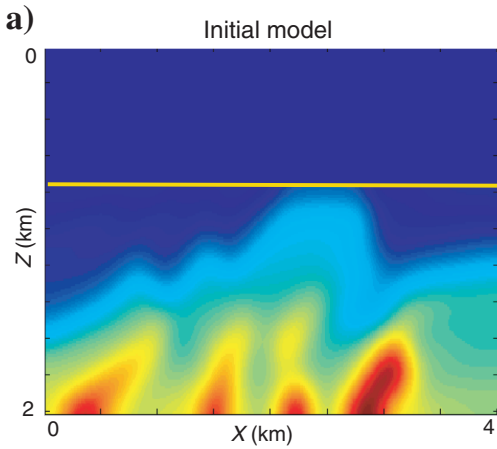

c)

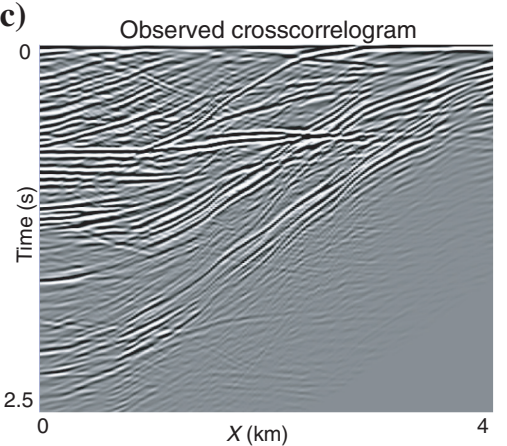

e)

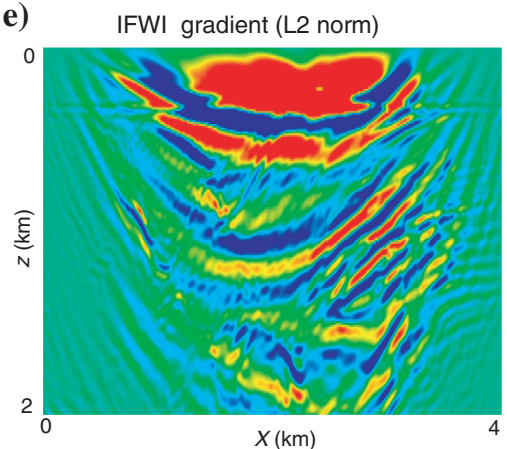

b)

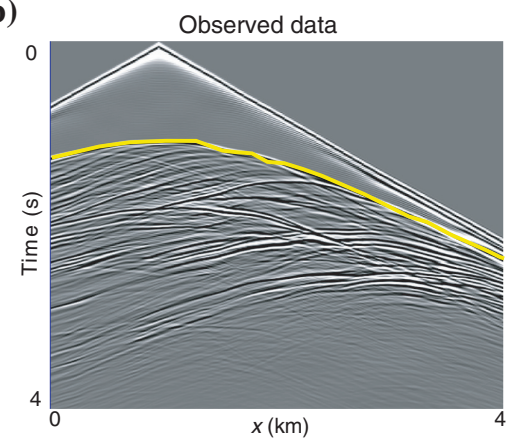

d)

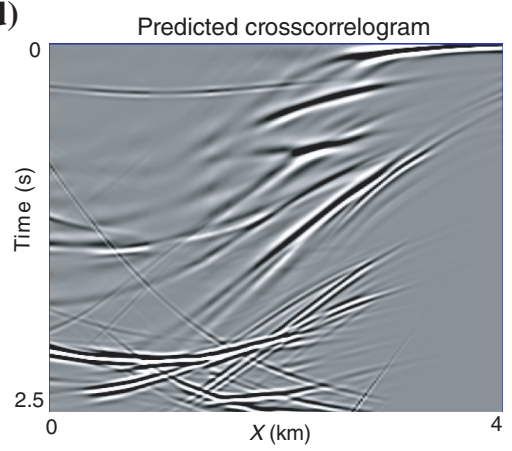

f)

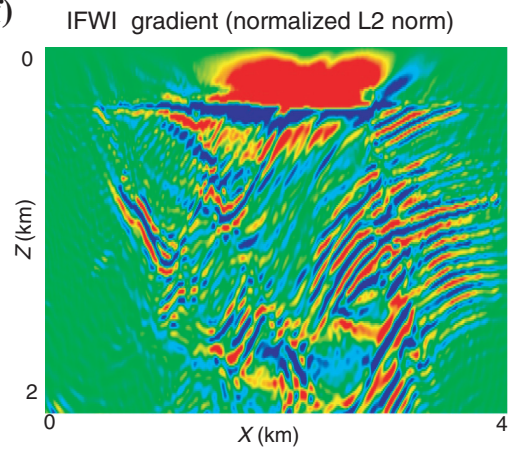




$$
\begin{gathered}
J(m)=\sum_{\omega} \sum_{s} \sum_{g} \frac{1}{2} \|\left(\widehat{\tilde{\Phi}}_{\text {mon }}(\mathbf{g} \mid \mathbf{s})-\widehat{\tilde{\Phi}}_{\text {base }}(\mathbf{g} \mid \mathbf{s})\right) \\
-\left(\hat{\Phi}_{\text {mon }}(\mathbf{g} \mid \mathbf{s}, m)-\hat{\Phi}_{\text {base }}\left(\mathbf{g} \mid \mathbf{s}, m_{0}\right)\right) \|^{2}
\end{gathered}
$$

\section{NUMERICAL RESULTS}

In the following examples, FWI and IFWI are implemented in the time domain using the trace-normalized $L_{2}$-norm misfit for the observed data and crosscorrelograms, respectively. A finite-difference solution to the second-order acoustic wave equation with secondorder accuracy in time and eighth-order in space is used for all the forward and backward propagation operations. Illumination compensation (Schleicher et al., 2008) estimated from the source-side
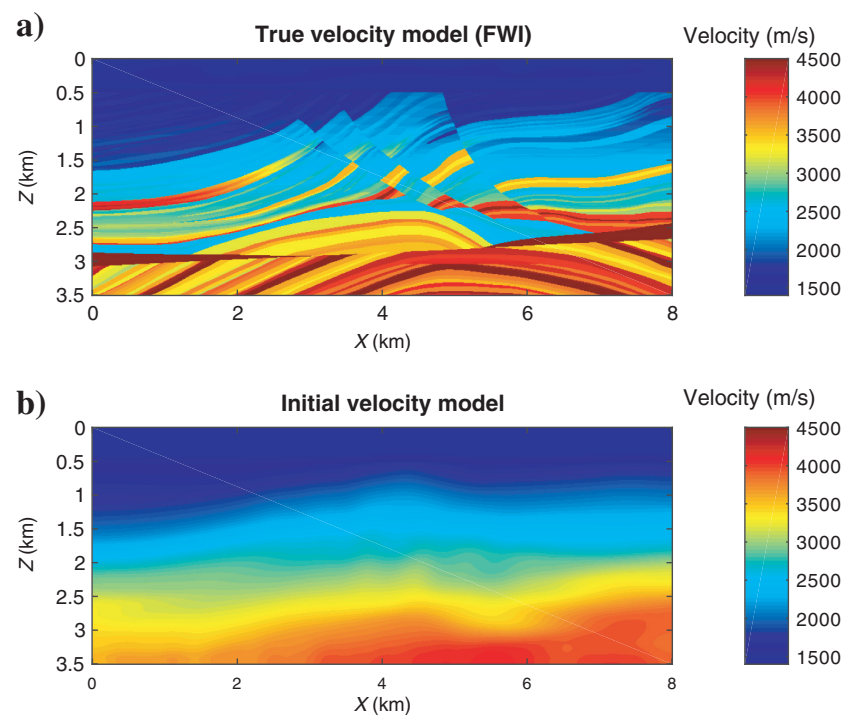

Figure 3. (a) Velocity model used for generating the observed data. The area inside the ellipse is ridden with velocity errors. (b) Initial velocity model for FWI and IFWI. The sea bottom (indicated in yellow) is used as the reference reflector.
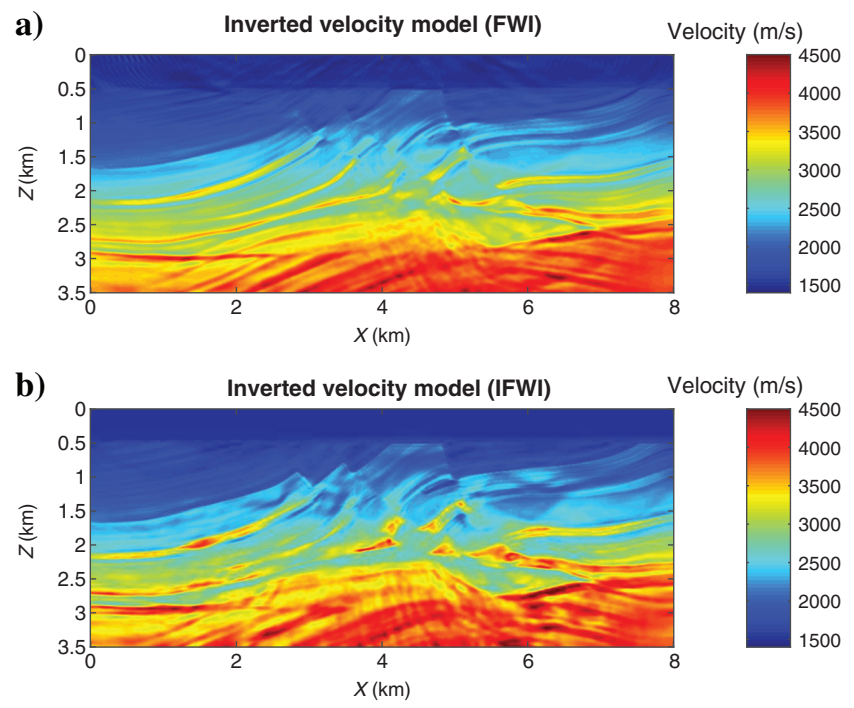

Figure 4. Inverted (a) FWI and (b) IFWI tomograms. wavefield is used as a preconditioner for all the inversions carried out.

\section{Marmousi model}

IFWI is tested on a modified version of the Marmousi model. A fixed spread acquisition geometry is used for generating the observed data. The seismic survey consists of 96 shots spaced at an interval of $80 \mathrm{~m}$, and there are 401 receivers spaced every $20 \mathrm{~m}$. Finite-difference solutions to the acoustic wave equation with absorbing boundary conditions are used to generate the observed data using the velocity model shown in Figure 3a. A $15 \mathrm{~Hz}$ Ricker wavelet is used for generating the data. The encircled region in Figure 3 a depicts the low-velocity anomalies added to the water layer, where the sea bottom is chosen as the reference reflector for IFWI and the starting model used for inversion is shown in Figure $3 \mathrm{~b}$.

The inverted velocity models after $50 \mathrm{CG}$ iterations of conventional FWI and IFWI are shown in Figure $4 \mathrm{a}$ and $4 \mathrm{~b}$, respectively.
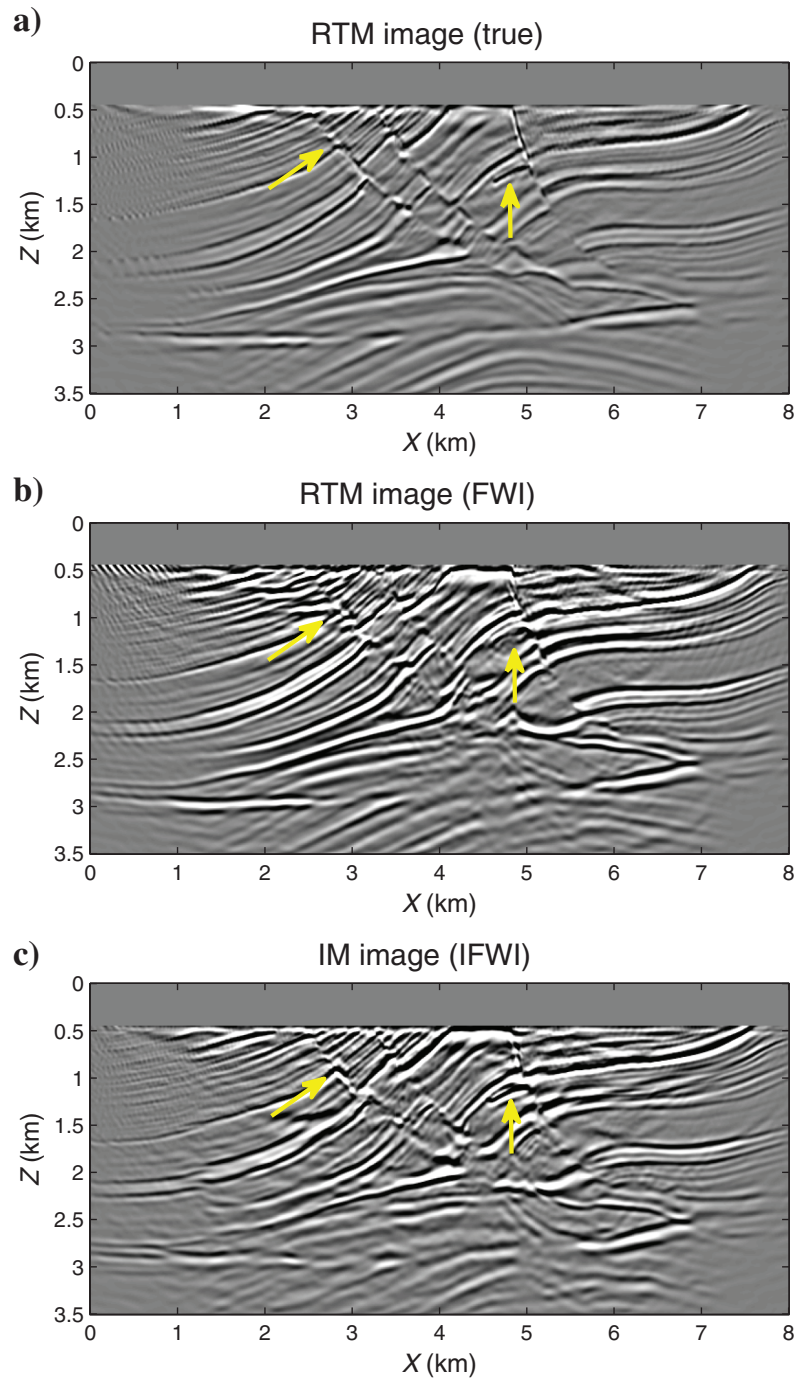

Figure 5. The RTM image with the (a) true model and (b) FWI tomogram as the migration velocity model. (c) IM image using the IFWI tomogram. Yellow arrows indicate the areas where reflectors are better imaged by the IFWI tomogram. 


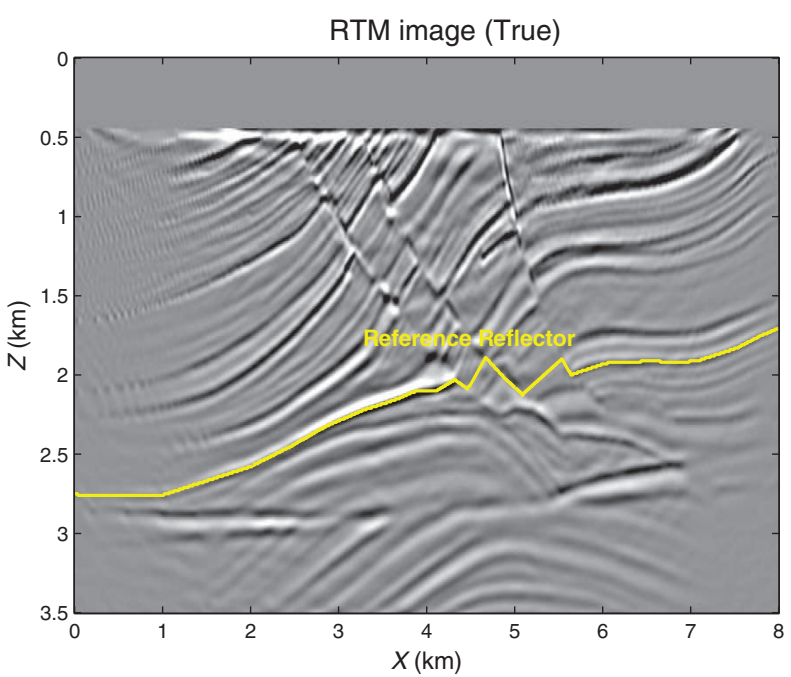

Figure 6. The new reference reflector is plotted in yellow on the migration image obtained from the true velocity model.

a)

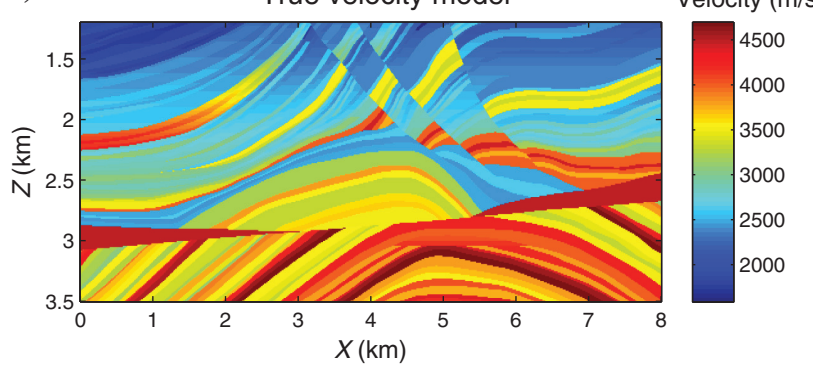

b)

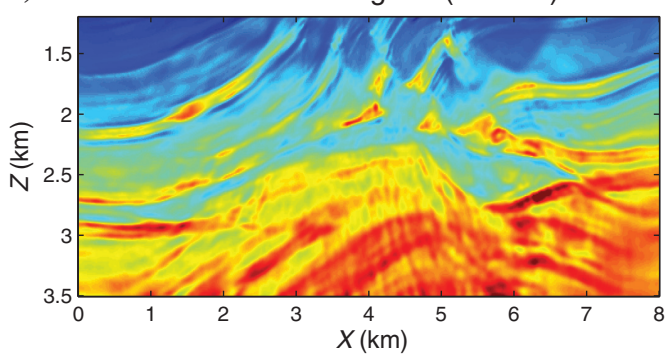

Velocity $(\mathrm{m} / \mathrm{s})$

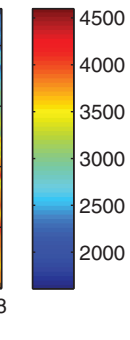

c)

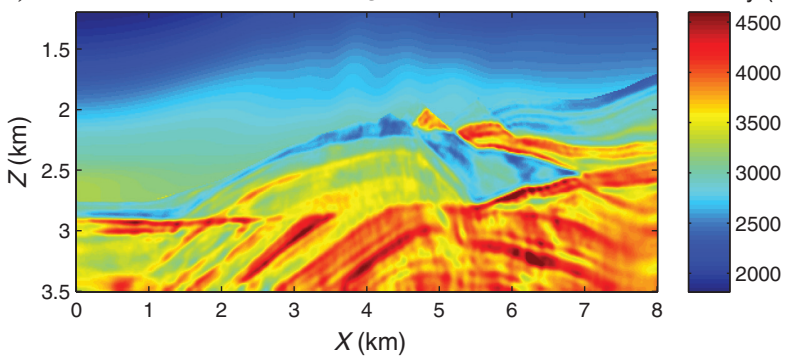

Figure 7. (a) True velocity model. Inverted IFWI tomograms using the (b) sea bottom and (c) a deeper reflector as reference reflectors, respectively.
The inverted tomogram using IFWI is more accurate than the one inverted by FWI in regions lying close to the reference reflector. This is because the gradient for IFWI is based on IM, which corrects for the unaccounted anomalies in the water column. In addition, the velocity model that is updated by IFWI is approximately 7 to 8 times the size of the FWI model, so IFWI will enjoy faster convergence than FWI for a nonlinear CG solver. However, the accuracy of the IFWI tomogram starts to suffer as we move further away from the reference interface as seen in the deeper areas. The FWI tomogram in shallow areas fails to recover the actual features of the model compared with its IFWI counterpart. To further support our claimed superiority of IFWI over FWI, we compare the migration images estimated using the two inverted tomograms with the true migration image. Figure 5a and 5b shows the migration images computed using the true and inverted FWI models, respectively. Figure 5c shows the IM image estimated using the IFWI tomogram. The reflection events marked by the yellow arrows indicate the areas where the IM image is in close agreement with the migration image estimated by the true velocity model compared with its FWI counterpart.

Now, we demonstrate the target-oriented application of IFWI for improving the velocity estimation in deeper regions by choosing a deeper reference reflector, as shown in Figure 6. The initial model is still the same as before, and 50 iterations of IFWI are used for the inversion. The inverted IFWI result is compared with the IFWI tomogram inverted using the sea bottom as a reference reflector in Figure 7. From Figure 7, it can be inferred that the velocity model is well-recovered in the deeper areas and is in good agreement with
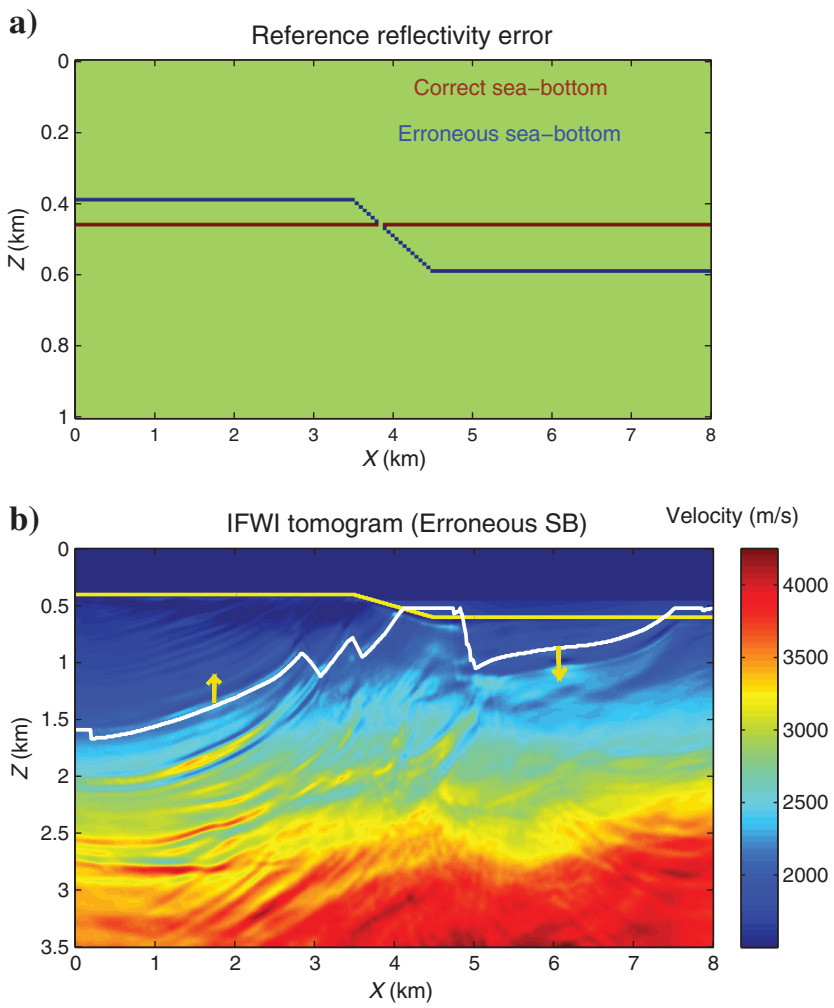

Figure 8. (a) Mispositioned sea-bottom reference reflector with the true sea bottom. (b) Inverted IFWI tomogram using the erroneous sea bottom as a reference reflector. The yellow arrows mark the translation of the recovered facies from their actual positions indicated by the white reflector obtained from the true model. 
the true model. Prior knowledge of a reflector in the subsurface will improve the accuracy of inverting for the subsurface velocity model below the reference interface.

\section{Sensitivity of IFWI to errors in the reference reflector}

Next, we evaluate how errors in the estimation of the reference reflector can lead to an erroneous IFWI tomogram. Instead of choosing the correct location of the sea bottom, we choose an erroneous sea-bottom estimate that is dislocated from its original position, as shown in Figure 8a. The distorted reference reflector is highlighted in blue in contrast to the true sea bottom indicated in brown.

a)

Initial velocity model

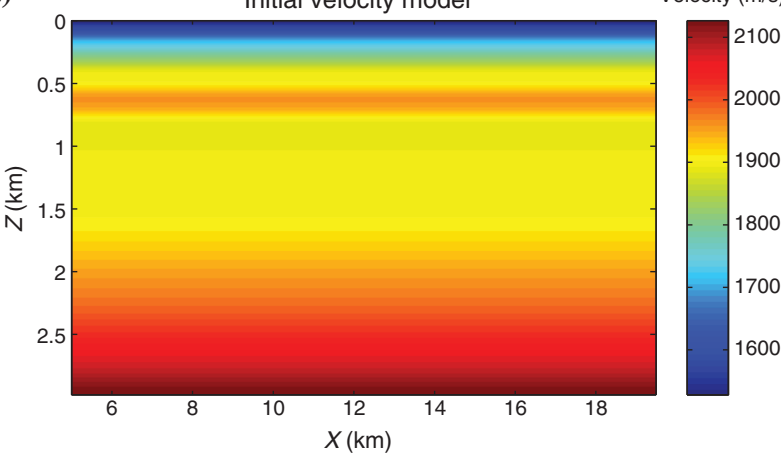

b)

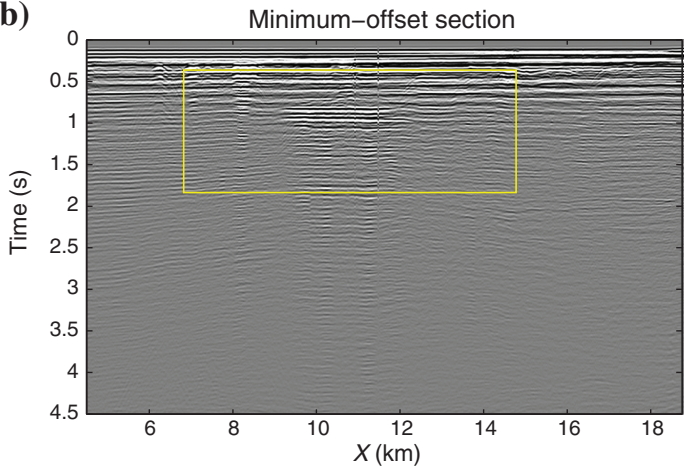

Figure 9. (a) Initial model inverted using plane-wave MVA. (b) The common-offset section for the source-receiver offset $=173 \mathrm{~m}$. The yellow box indicates the area where static shifts can be observed.

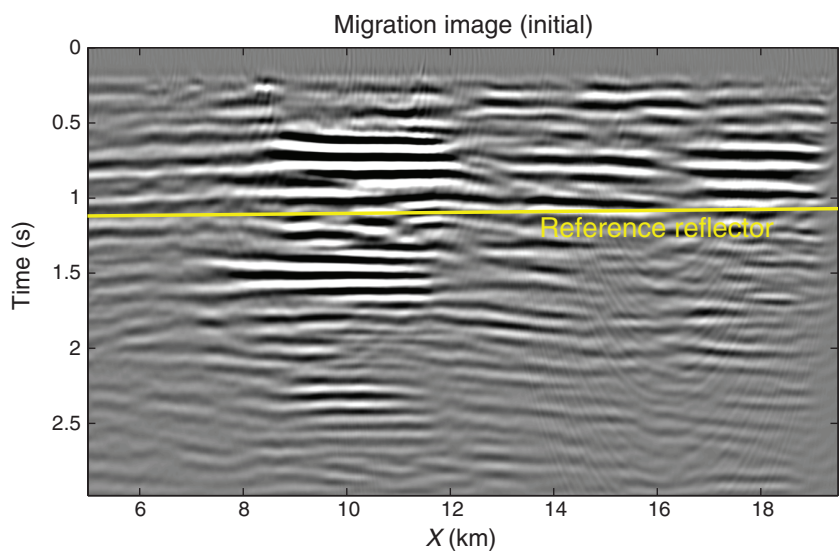

Figure 10. Migration image calculated using the initial velocity model with the assumed reference reflector indicated in yellow.
Using an incorrect reference reflector model leads to erroneous estimates of the subsurface velocity model below the reference interface, thereby resulting in misplacement of reflectors. If the incorrect choice of the reference-reflector location is far enough from the
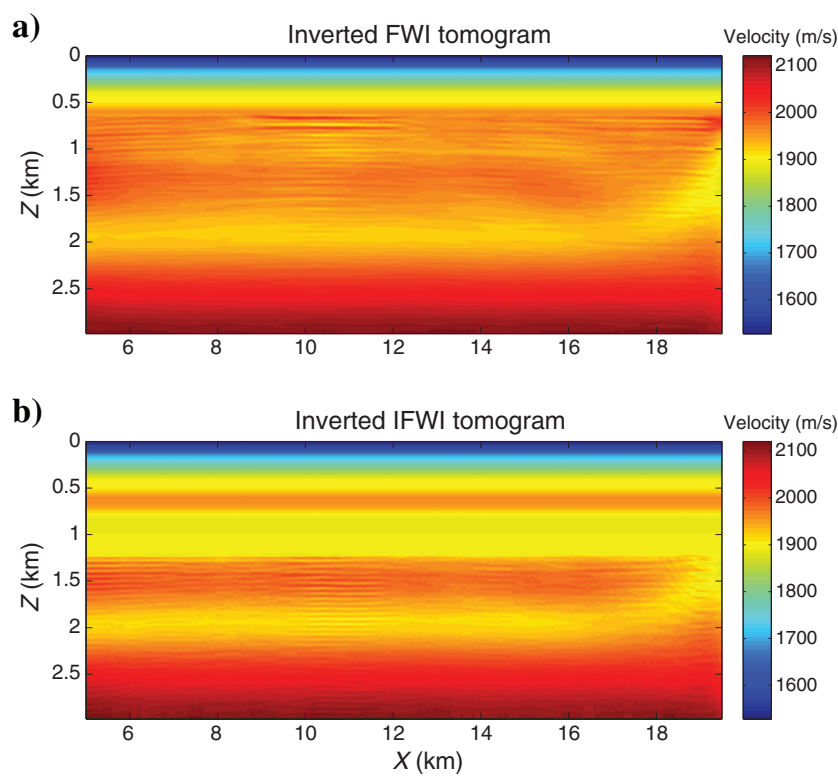

Figure 11. (a) FWI and (b) IFWI tomograms.

a)

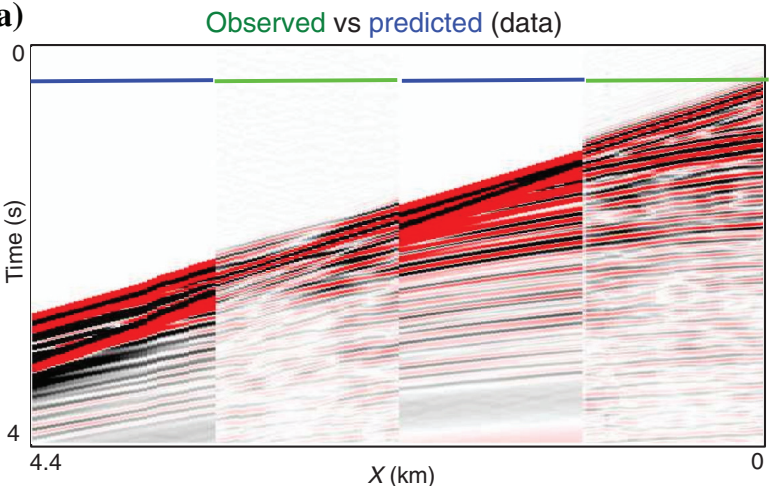

b)

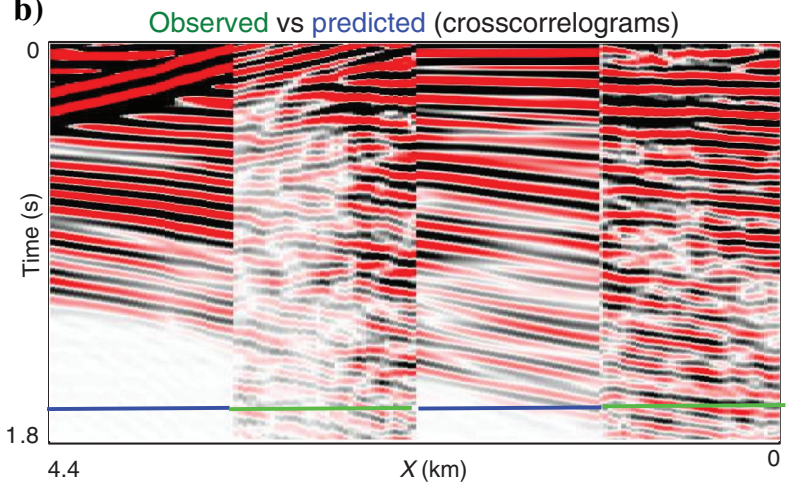

Figure 12. Interleaved panels of (a) observed and predicted data estimated using the inverted FWI tomogram in Figure 11a and (b) observed and predicted crosscorrelograms computed by the IFWI tomogram in Figure 11b. The observed and predicted panels are highlighted by green and blue lines, respectively. 
true reflector to cause the time differences between the observed and predicted crosscorrelograms to exceed half the dominant period, then there will be errors in the tomogram due to cycle skipping. This phenomenon is observed in Figure 8b, where the inversion gets stuck in a local minimum and the velocity values are not accurately recovered. As indicated by the yellow arrows, the facies in the recovered model are misplaced from their true positions defined by the white outline. Toward the left side, the events are placed shallower than their actual locations due to the underestimation in the depth of the reference reflector. On the contrary, facies in the right side of the model are shifted downward owing to the overestimation of the reference reflector depth.

\section{Field data test 1}

A marine data set was recorded in the Gulf of Mexico and 571 shot gathers were the input data with shots spaced every $25 \mathrm{~m}$ along the towed streamer. Each shot gather is recorded by 177 hydrophones, with a receiver spacing of $25 \mathrm{~m}$ and a maximum source-receiver offset of $4648 \mathrm{~m}$. A record length of $4.5 \mathrm{~s}$ was used for the inversion. The initial model shown in Figure 9a is obtained by plane-wave migration velocity analysis (Guo and Schuster, 2017), where the data suffer from static errors caused by lowvelocity anomalies in shallow gassy muds (Sheng et al., 2006). This can also be seen by observing the minimum-offset section (offset of $173 \mathrm{~m}$ ) in the data shown in Figure 9b, where the yellow rectangle

a)

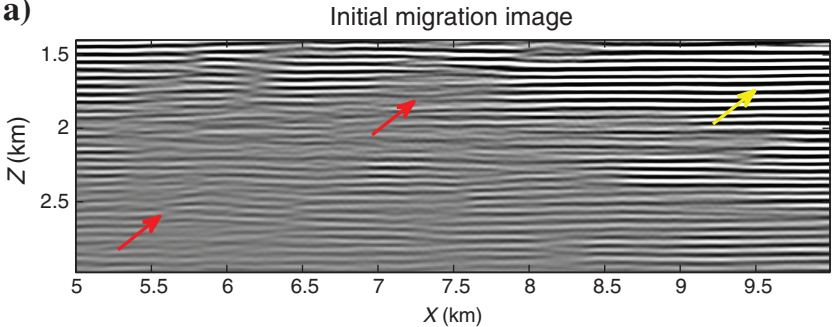

b)

FWI migration image

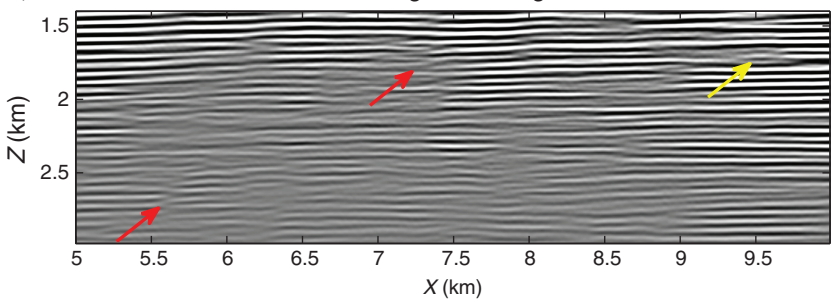

c)

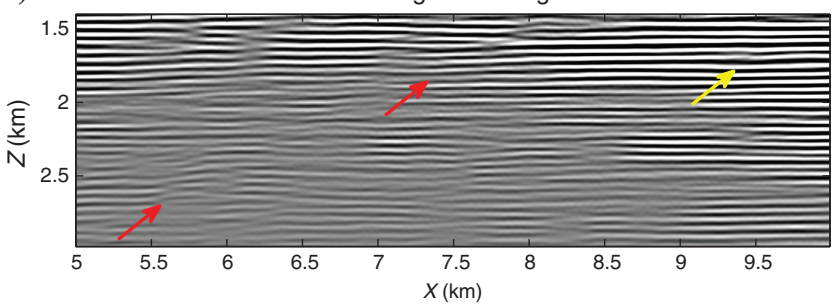

Figure 13. Migration images for zone A calculated using the (a) initial, (b) inverted FWI, and (c) IFWI tomograms as the migration velocity model. The red arrows indicate areas where IFWI performs better than FWI, and the yellow arrows mark the places where FWI performs worse than the initial and IFWI tomograms. indicates the area where velocity pull-down effects can be observed due to a localized low-velocity anomaly.

The data used for FWI and IFWI have a dominant frequency of $10 \mathrm{~Hz}$. For IFWI, we select a reference reflector, as shown in Figure 10. The selected reference interface is assumed to be flat because the zero-offset section mostly consists of flat events. The reflector in the initial migration image is windowed around the flat reflector, and Born modeling is used to generate observed reference data for IFWI. The inverted FWI and IFWI results are shown in Figure 11a and $11 \mathrm{~b}$, respectively. The IFWI updates are masked above the reference interface depth level as discussed in the theory section, but the FWI result was masked in the very shallow part only. Figure 12 compares the predicted and observed traces for FWI and IFWI. The observed and predicted panels are represented by green and blue markers, respectively. The predicted data computed from the FWI tomogram mostly agrees with the observed data, as shown in Figure 12a. The predicted crosscorrelograms estimated from the inverted IFWI tomogram and observed crosscorrelogram are well-aligned in most places, but there are slight misalignments in some places, which might be attributed to errors in the geometry of the assumed reference reflector.

To assess the fidelity of the inverted FWI and IFWI tomograms, we next compare the migration images and common-angle gathers. For IFWI, the migration images are estimated using IM as seen in the previous synthetic example. The data used for migration are band-passed to have a dominant frequency of $20 \mathrm{~Hz}$. We divide the

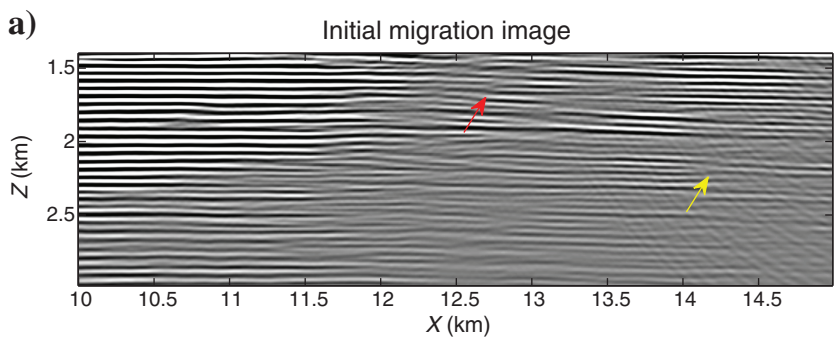

b) $\quad$ FWI migration image

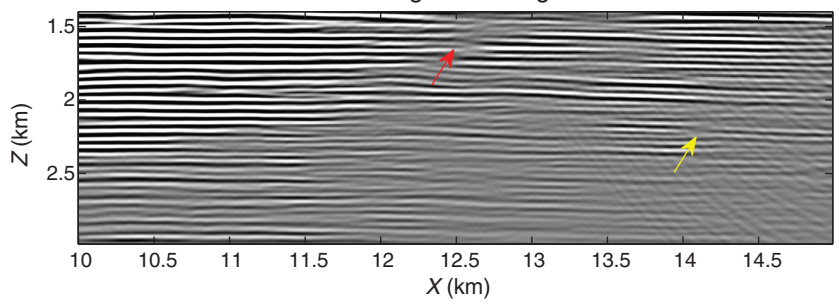

c)

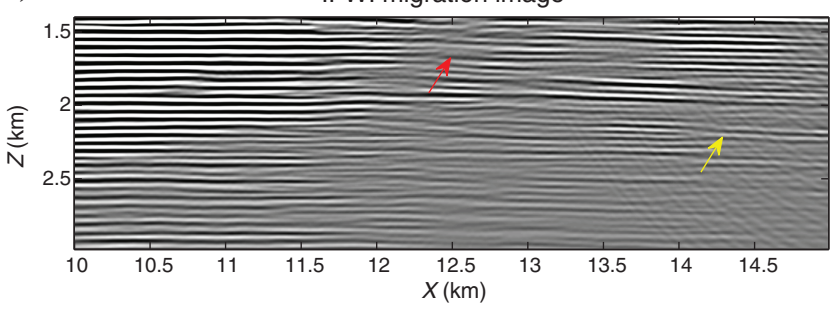

Figure 14. Migration images for zone B calculated using the (a) initial, (b) inverted FWI, and (c) IFWI tomograms as the migration velocity model. The red arrows indicate areas where IFWI performs better than FWI, and the yellow arrows mark the places where FWI performs worse than the initial and IFWI tomograms. 
migration images into two zones, A and B. Figures 13 and 14 show the migration images for zones $\mathrm{A}$ and $\mathrm{B}$, respectively. The reflectors in the IFWI migration images for zones $\mathrm{A}$ and $\mathrm{B}$ are more coherent and better focused in comparison with its FWI and initial image counterparts as indicated by the red arrows. The regions marked by yellow arrows indicate areas where the FWI migration image has deteriorated in comparison with the IFWI and initial migration images. These observations support the claim that the FWI tomogram is affected by the absence of unresolved low-velocity anomalies in the shallow part of the subsurface.

Figure $15 \mathrm{a}-15 \mathrm{c}$ shows the common-angle gathers using the initial, inverted FWI, and IFWI models, respectively. FWI slightly improves the velocity model over the initial model, but in the $X=10 \mathrm{~km}$ panel, IFWI common-angle gathers appear much flatter and coherent than their FWI counterparts.

Figure 15. Common-angle gathers computed using the (a) initial model shown in Figure 9a, (b) FWI tomogram shown in Figure 11a, and (c) IFWI tomogram shown in Figure 11b. Yellow rectangles mark the areas where reflection events are flatter in the CIGs obtained from IFWI. a)

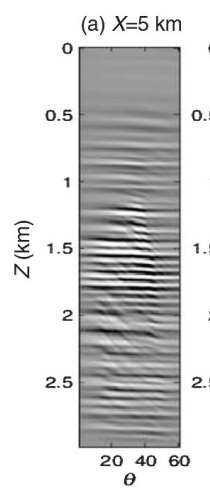

b)

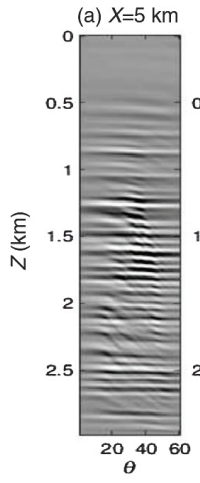

c)

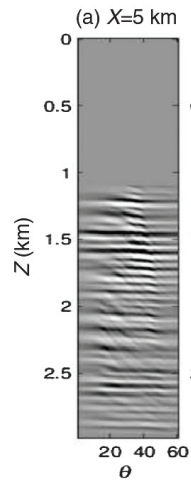

\section{Field data test 2}

This marine data set was also recorded in the Gulf of Mexico with minimum and maximum offsets of 200 and $6200 \mathrm{~m}$, respectively. The streamer data consist of 496 shot gathers shot at an interval of $37.5 \mathrm{~m}$, where each shot was recorded using 480 hydrophones spaced at an interval of $12.5 \mathrm{~m}$. The MSFWI tomogram inverted from a previous study (Huang, 2013) is shown in Figure 16a, where the reflector marked in yellow is chosen as the reference reflector. Early arrivals and reflection data were used to generate this tomogram, but for the current example only reflections are used. The initial model used for inversion is shown in Figure 16b, and velocity variations are added to the water column, as shown by the yellow rectangles. Based on the magnitude spectrum of the data shown in Figure 17, the inversion is carried out in two stages centered around the two dominant frequencies (approximately 9 and $20 \mathrm{~Hz}$ ). The
Initial angle gathers

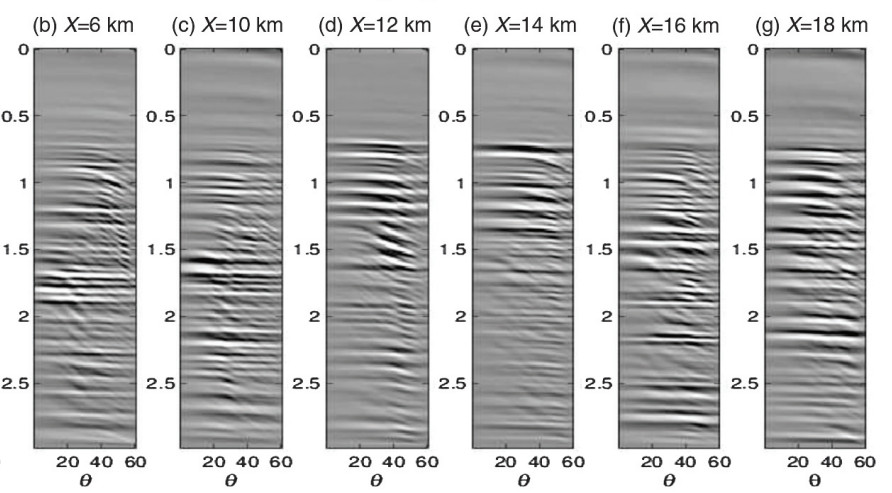

$\mathrm{FWl}$ angle gathers

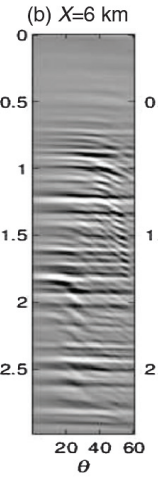

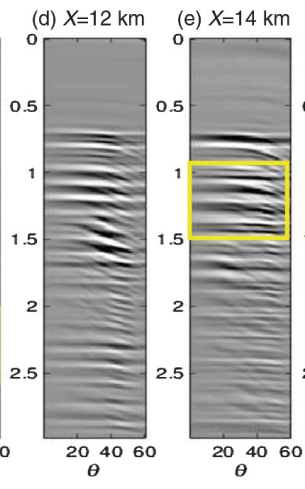

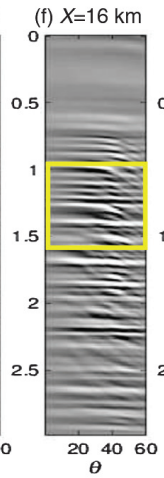

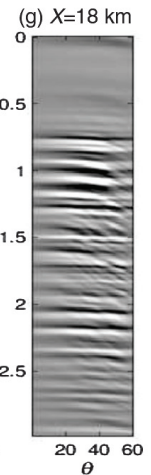

IFWI angle gathers
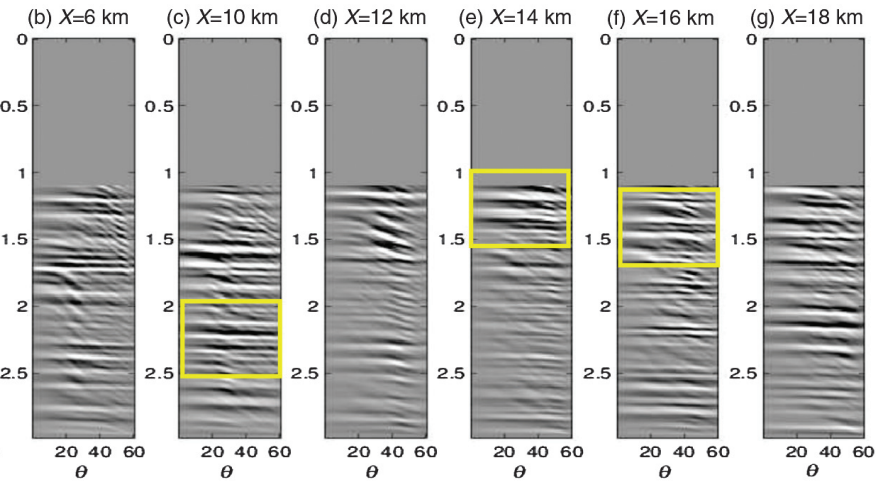
data were filtered for the two multiscale inversion schedules using Wiener filtering to estimate the matching filters for Ricker wavelets with central frequencies of 9 and $20 \mathrm{~Hz}$, respectively. The velocity errors of $50-100 \mathrm{~m} / \mathrm{s}$ have been added to the initial model to demonstrate the efficacy of IFWI in countering the overburden velocityestimation errors.

a)

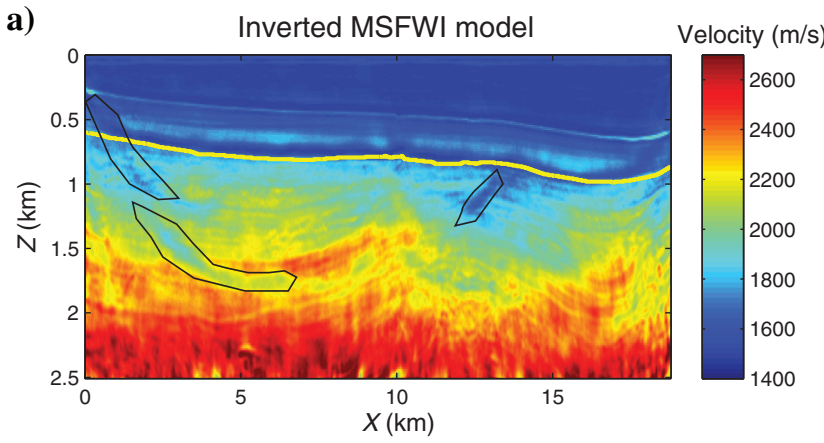

b) Initial model with water-column statics

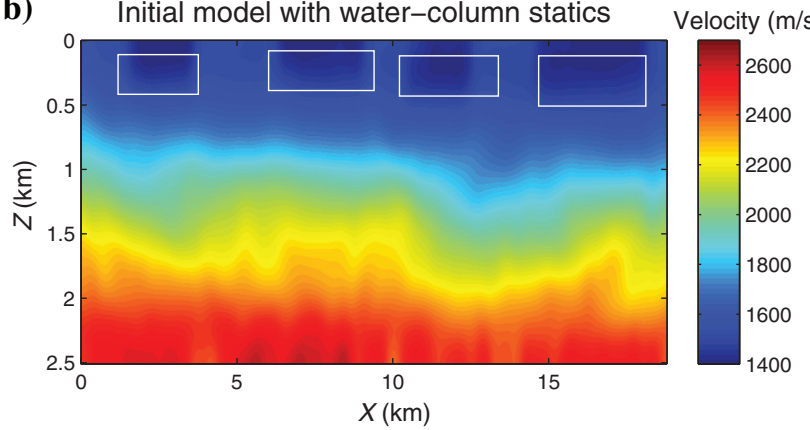

Figure 16. (a) The FWI velocity model used for selecting the reference reflector (yellow) and (b) initial velocity model with added errors (indicated by white boxes) in the water column. The encircled areas represent some low anomalies in the tomogram, which seem to coincide with the diving wave raypaths, these are probably caused by cycle skipping of some of the diving waves.

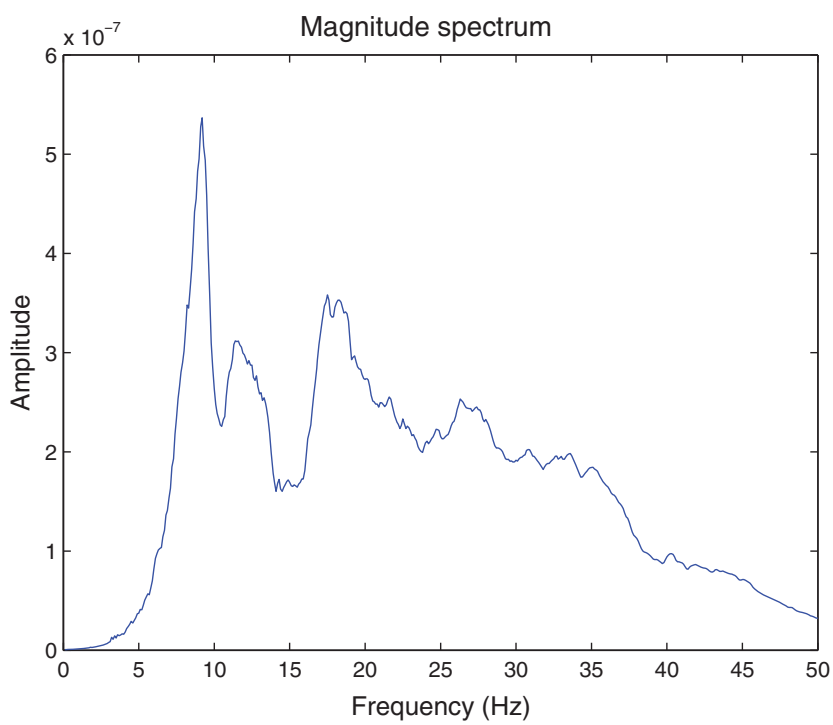

Figure 17. Amplitude spectrum of a common shot gather shows two dominant peaks at approximately 9 and $20 \mathrm{~Hz}$.
These errors are sufficient to cause the FWI to get stuck in a local minima as seen in Figure 18a, whereas IFWI manages to overcome the water column errors and provides a more resolved tomogram shown in Figure 18b. This is also illustrated by Figure 19, after five iterations FWI gets stuck in a local minima, whereas the IFWI crosscorrelogram residual drops to $30 \%$ of the initial residual. The interleaved data and crosscorrelogram are shown in Figure 20, where the
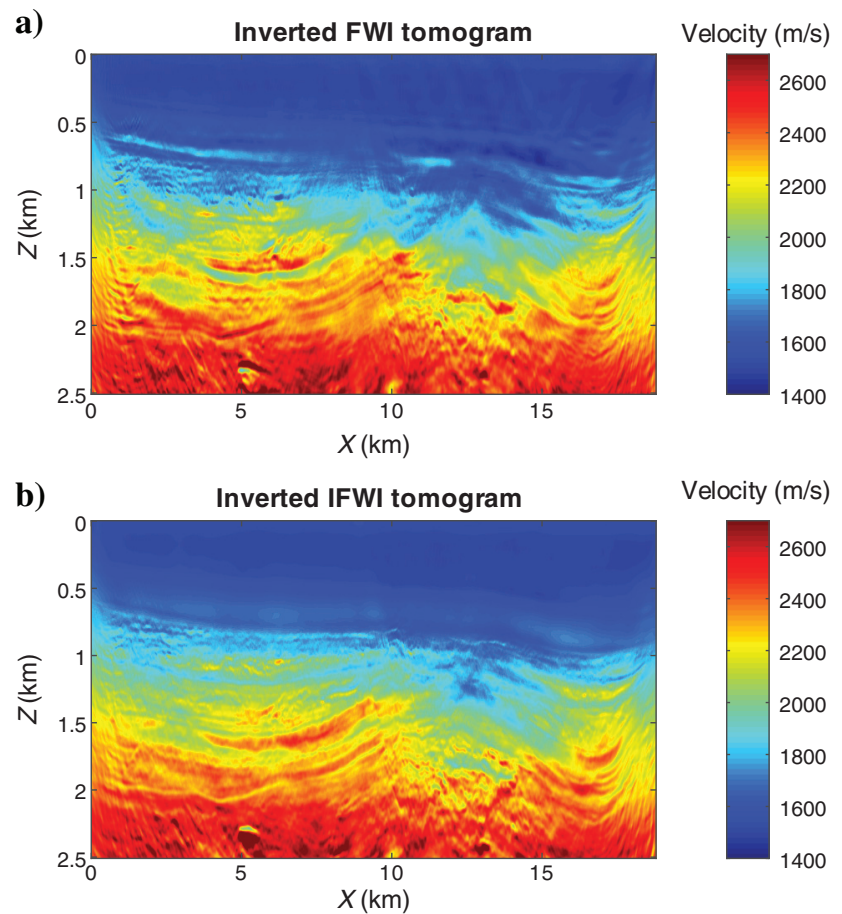

Figure 18. (a) The FWI and (b) IFWI tomograms.

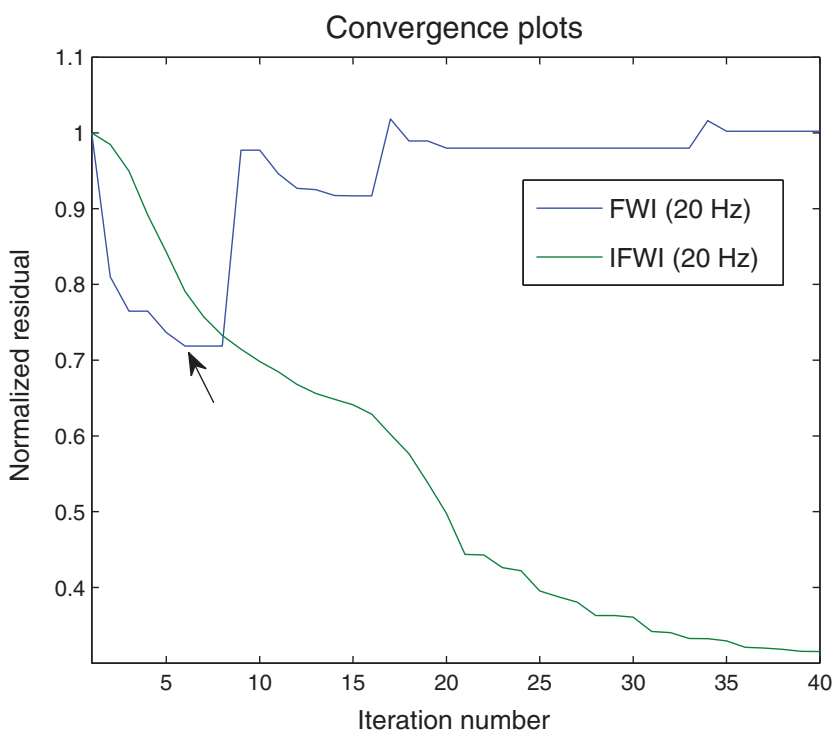

Figure 19. Convergence curves corresponding to the final schedule $(20 \mathrm{~Hz})$ of inversion for FWI (blue) and IFWI (green); the black arrow corresponds to the local minima FWI tomogram shown in Figure 18a. FWI and IFWI misfit values have been normalized by their respective first iteration misfit values. 
observed and predicted crosscorrelogram largely agree with one another. On the other hand, the predicted data computed from the FWI tomogram are unable to match the observed data. This is because the errors in the water column cause the predicted data to cycle skip. Figure 21a highlights this problem, where for the given range of offsets, the discrepancy between the observed and predicted wiggles grows with the offset, whereas the observed and predicted wiggles in the crosscorrelograms are well-aligned with each other. The black ellipses in Figure 21a and 21b highlight reflection events where the cycle skipping is attenuated relative to the FWI traces.

The migration image computed using the FWI tomogram shown in Figure 16a is used as a reference for assessing the quality of the IM image computed from the IFWI tomogram. Figures 22 and 23 show the migration images for the two halves of the survey, where the inverted IFWI migration image is in close agreement with the reference migration image. In Figure 22a, the yellow box shows a likely artifact in the reference migration image caused by false lowvelocity anomalies in Figure 16a tomogram. These anomalies are probably caused by cycle skipping of diving waves because for IFWI, only reflections are used; we do not observe these artifacts in the tomogram and the migration image.

\section{Time-lapse synthetic}

IFWI is tested on a 2D model based on geology of the Gulfaks field in Norway (Raknes and Arntsen, 2014). A fixed-spread
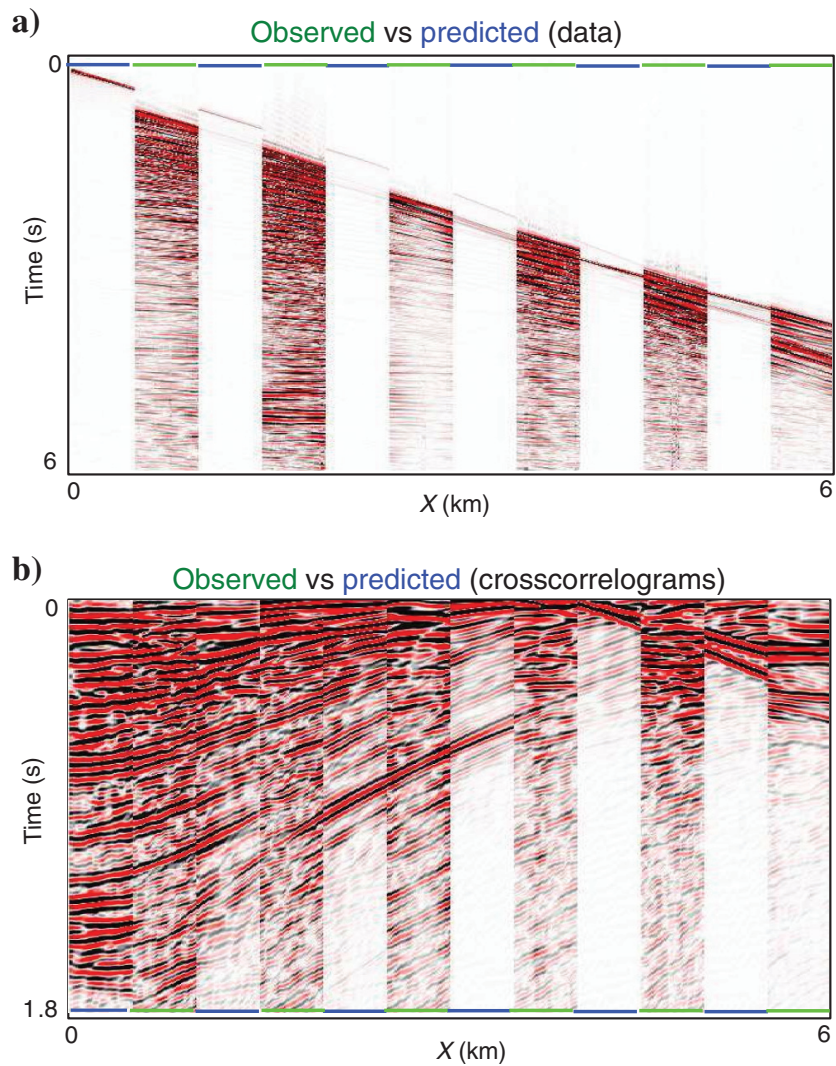

Figure 20. Interleaved panels of (a) observed and predicted data estimated using the inverted FWI tomogram in Figure 18a and (b) observed and predicted crosscorrelograms computed by IFWI tomogram in Figure 18b. The observed and predicted panels are highlighted by green and blue lines, respectively. acquisition survey is used to generate the baseline data. The survey consists of 96 shots spaced at an interval of $60 \mathrm{~m}$ at a depth of $10 \mathrm{~m}$ below the sea surface. Each shot gather is recorded by a line of 301 receivers with receivers spread at an interval of $20 \mathrm{~m}$. The velocity models used for generating the baseline data and time-lapse changes in the velocity are shown in Figure $24 \mathrm{a}$ and $24 \mathrm{~b}$, respectively. The water-column depth extends down to $1 \mathrm{~km}$ to replicate a deepwater scenario. To simulate nonrepeatability in the monitor data, small variations on the order of $20-40 \mathrm{~m} / \mathrm{s}$ are introduced in the water layer, and the shot locations are randomly shifted to simulate tidal variations, as shown in Figure 24b. The reservoir lies at the $2 \mathrm{~km}$ depth

a)

\section{Observed vs predicted} near-offset data

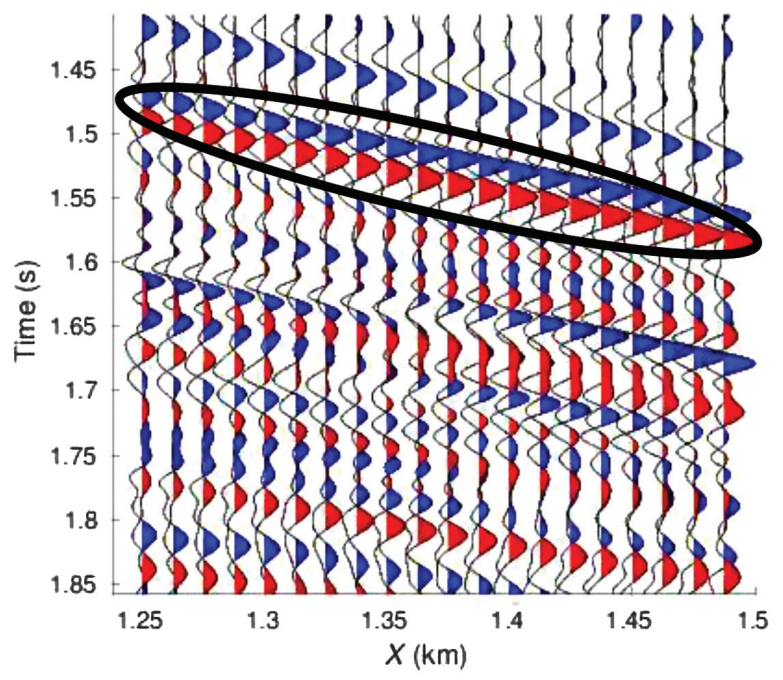

b)

Observed vs predicted near-offset crosscorrelograms

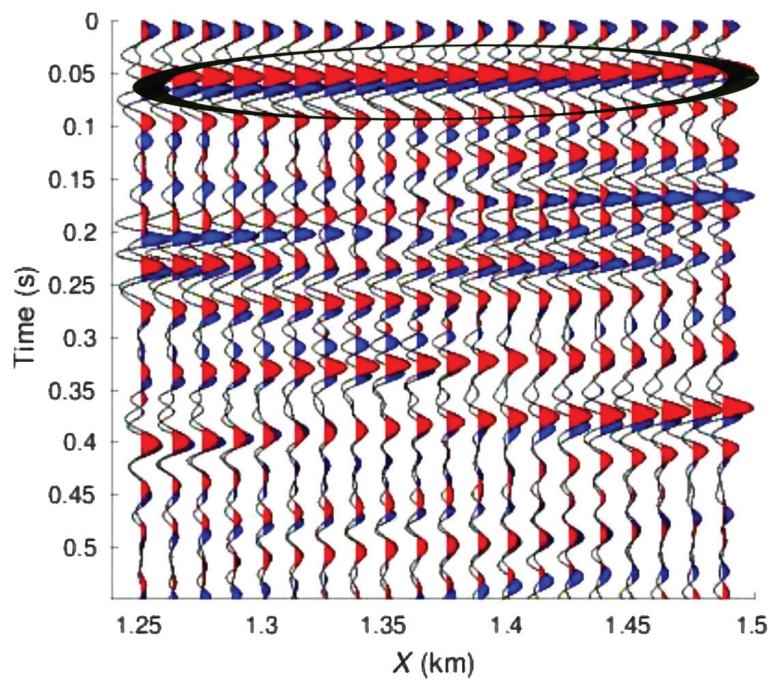

Figure 21. Predicted (blue) and observed (red) waveforms for a common shot gather are compared in the (a) data and (b) crosscorrelogram domains. These waveforms have been computed from the 10th iteration FWI and IFWI tomograms for $20 \mathrm{~Hz}$ schedule. The black ellipse in (a) marks a reflection event, which is cycle skipped in the data domain, but the cycle skipping is nullified for the same event in (b). 
level, as shown in Figure 24b. A second-order in space and eighthorder in time finite-difference scheme with an $8 \mathrm{~Hz}$ Ricker wavelet as the source signature is used to generate both data sets. For simplicity, the baseline and monitor surveys are referred to as years 1 and 2, respectively. Only reflections are inverted for both surveys. We use the double-difference strategy discussed previously for inverting the time-lapse change in the monitor data set.

Figure 25a and 25c shows the FWI and IFWI tomograms for year 1 , respectively. Now, we use DDFWI and DDIFWI to mitigate the spurious artifacts that are characteristic of the nonlinear inversion
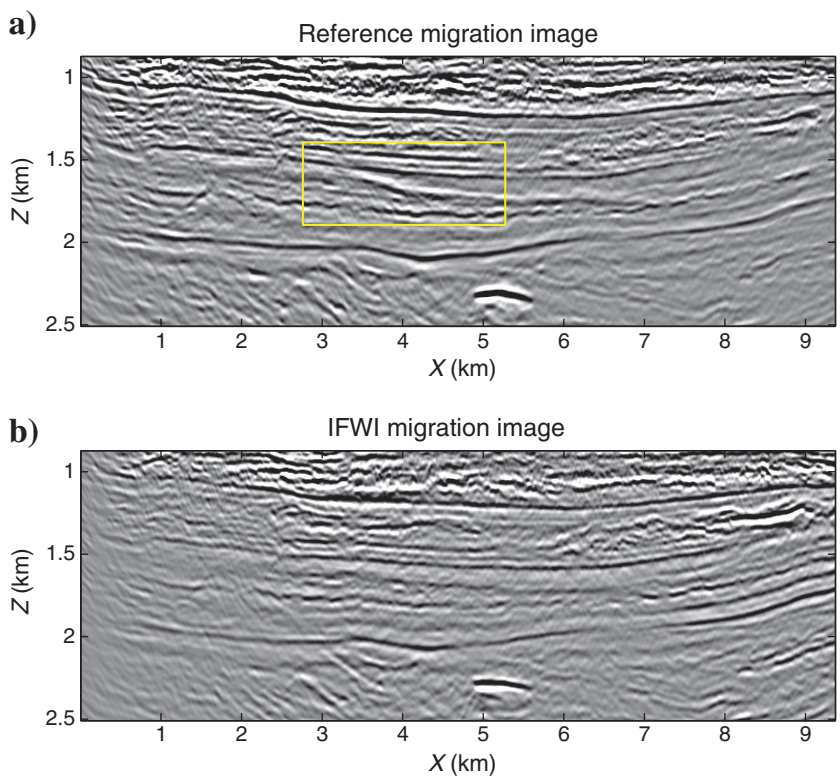

Figure 22. (a) Reference and (b) IFWI migration images for the first half of the survey. The yellow box shows the artifact seen in the reference migration image, which is mitigated in the image estimated from the IFWI tomogram.
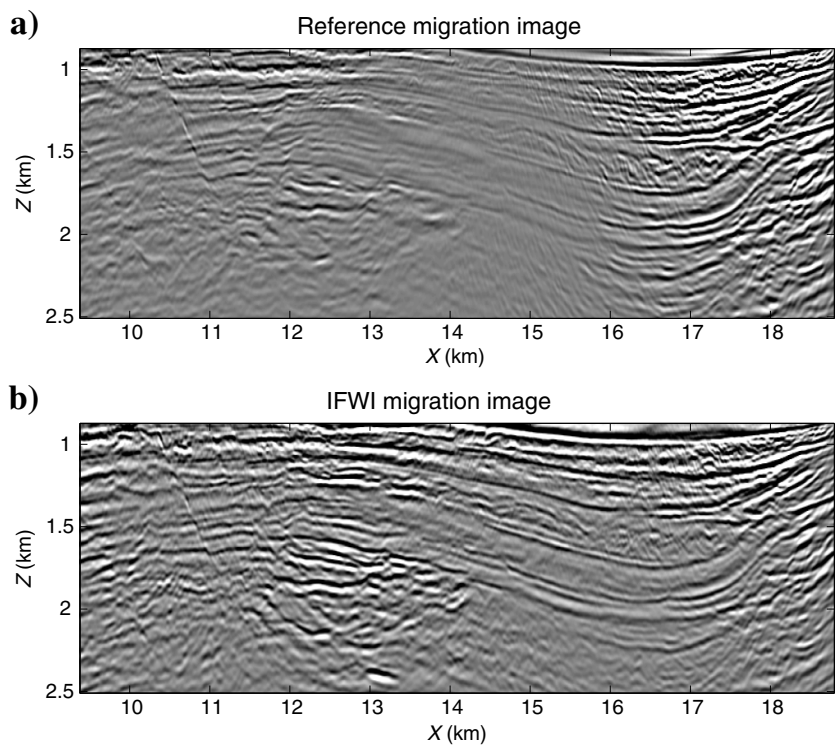

Figure 23. (a) Reference and (b) IFWI migration images for the latter half of the survey. method. The FWI and IFWI tomograms inverted from the baseline surveys are used as initial models for inverting the year 2 DDFWI and DDIFWI tomograms shown in Figure 25b and 25d. The timelapse changes are computed by subtracting the monitor DDFWI and DDIFWI tomograms from their inverted baseline FWI and IFWI counterparts. Figure 26 shows the time-lapse changes computed by DDFWI and DDIFWI.

DDIFWI highlights changes mostly at the reservoir level, whereas the DDFWI estimated time-lapse changes indicate variations at not only the reservoir but also in the nonreservoir areas where no actual changes occurred. These spurious changes are caused by nonrepeatability errors added to the data. The doubledifference approach combined with IFWI mitigates the spurious artifacts caused by overburden variations above the reference reflector, and it provides an accurate estimate of the time-lapse changes in the reservoir area. To further validate the reduction of artifacts in IFWI tomograms, we inspect the final double-difference data and crosscorrelogram misfits for DDFWI and DDIFWI. Figure 27b shows that the double-difference crosscorrelogram residual for the most part has been nullified except for a spurious deeper event. For comparison, the values computed by DDFWI in Figure 27a are still persistent because of the nonrepeatability discrepancies.

\section{DISCUSSION}

The tests on synthetic and field data reveal that IFWI can be used for recovering an accurate velocity model several wavelengths below the reference reflector. The main factor driving the accuracy of the method is the depth of the reflectors relative to the reference interface. Areas lying closer to the reference interface are imaged more accurately. As shown in the Marmousi and field-data examples, IFWI was able to invert for a more accurate tomogram in the deeper target area. Figure 28 shows the well-log comparison with the FWI, IFWI (seabottom), and IFWI (deep) tomograms associated with the Marmousi model. In blue, the shallow areas IFWI with the
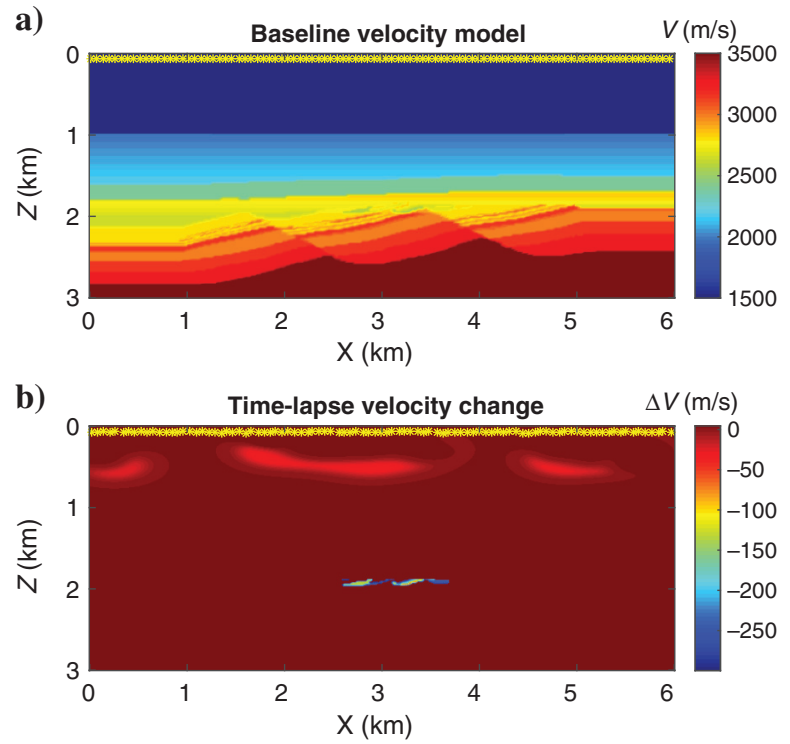

Figure 24. (a) Baseline velocity model and (b) time-lapse changes between the two surveys with shot locations indicated by yellow stars. 
sea bottom performs well, but the velocity errors increase with depth. The well-log comparison shown in Figure 28e and 28f shows an improved recovery of velocity values when the deeper reference reflector is chosen as a reference reflector for IFWI.

This feature of IFWI makes it suitable for target-oriented waveform inversion. In addition, fewer iterations are needed to achieve convergence compared with FWI. The high-wavenumber components of the FWI gradient are formed by ellipses arising from the migration of the reflections. The gradient of IFWI is based on IM, which by virtue of the reference interface produces the ellipses at a more accurate location compared with the FWI gradient. This is demonstrated in Figure 29b, which shows the IFWI gradient at the first iteration has better high-wavenumber updates in shallow areas close to the reference interface compared with its FWI counterpart in Figure 29a. By correctly placing the reflectors after the first iteration, the rabbit-ear wavepaths with intermediate wavenumber updates are formed at the correct locations in the subsurface in the following iterations.

Natural redatuming by IFWI is rooted in the assumption that raypaths for the reference reflection coincide with those for the underlying reflections, but this assumption is violated as we move away from the reference reflector. This leads to an increase in datuming errors with deeper reservoirs. To overcome this challenge, we can iteratively choose deeper reference reflectors so that reservoir changes can be evaluated accurately. Examples of this are shown in Sinha and Schuster (2016). This could be important for onshore data where overburden errors are more pronounced because of the complex near surface (Jones, 2012). An accurate IFWI tomogram can be computed using a reference reflector below the complex near surface, so that the near-surface errors can be bypassed. IFWI might also be significant for deepwater imaging because the water column is larger in size, so there is a greater scope for variations in the water-layer velocity. A layer-stripping approach could be used in conjunction with IFWI for inverting deeper sections of the subsurface. This can be done by dividing the subsurface below the overburden into several target zones bounded by prominent reflectors and inverting these target areas one at a time. By inspecting the common-angle image gathers after each set of inversion, a new reference interface can be chosen for inverting the subsequent target zone until the complete model has been inverted.

We also test the feasibility of time-lapse IFWI to account for the nonrepeatability errors caused by the time-varying overburden over the course of different experiments. The results from the synthetic example show that IFWI coupled with the double-difference approach can recover the time-lapse changes more accurately than FWI. For the examples shown here, the sea-bottom reflector was chosen as a reference, and it is assumed that the location of the sea bottom is known accurately for the monitor and baseline surveys.
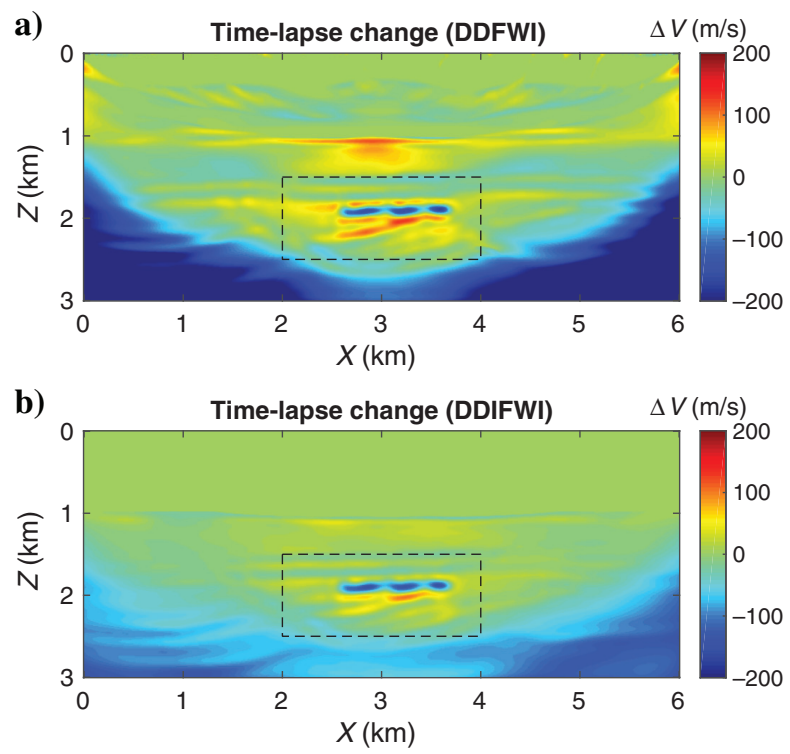

Figure 26. Time-lapse tomograms computed by (a) DDFWI and (b) DDIFWI.
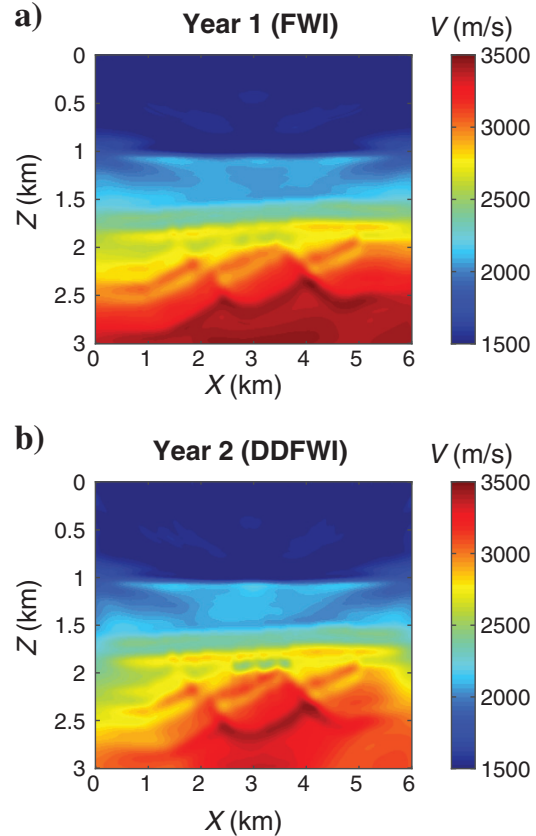

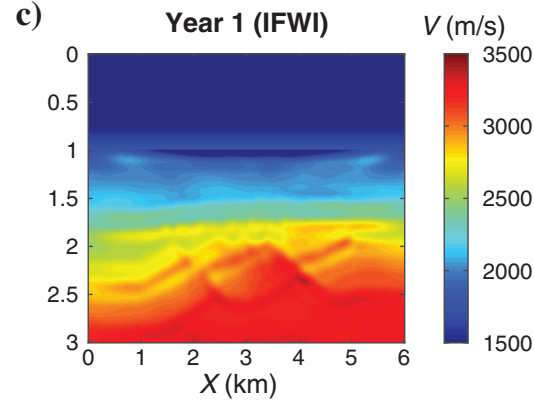

d)

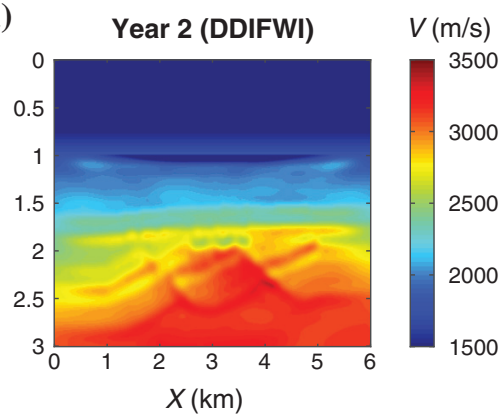

Figure 25. Inverted (a) FWI baseline, (b) DDFWI monitor, (c) IFWI baseline, and (d) DDIFWI monitor tomograms. 
a)

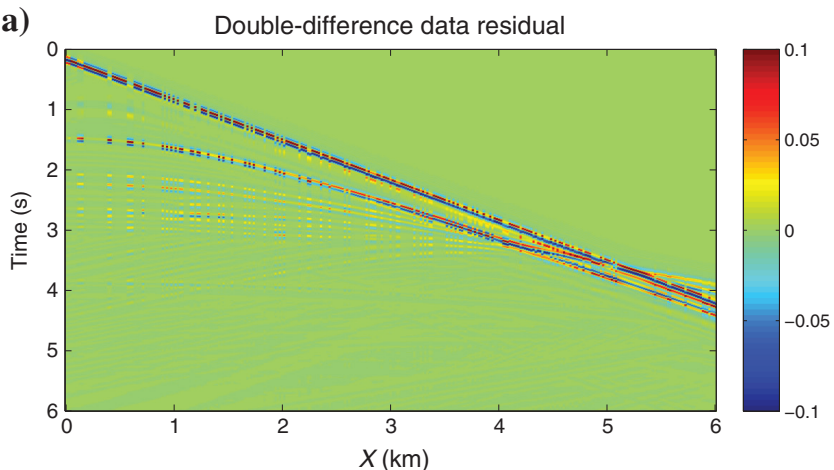

b) Double-difference crosscorrelogram residual

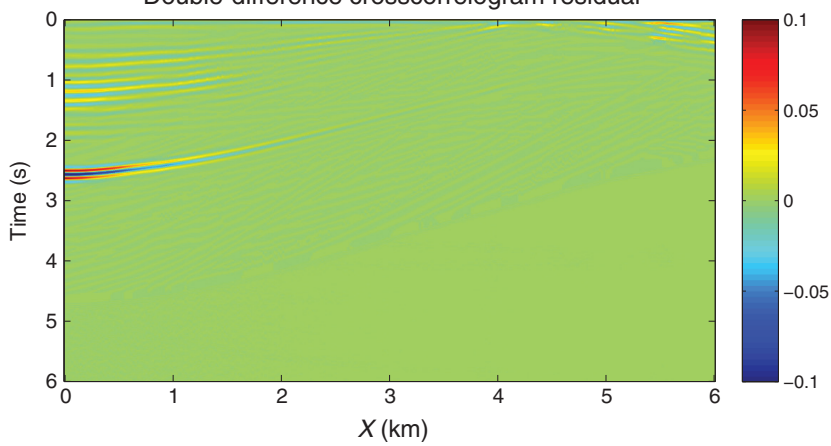

Figure 27. Double-difference residuals for (a) DDFWI and (b) DDIFWI misfits. a)

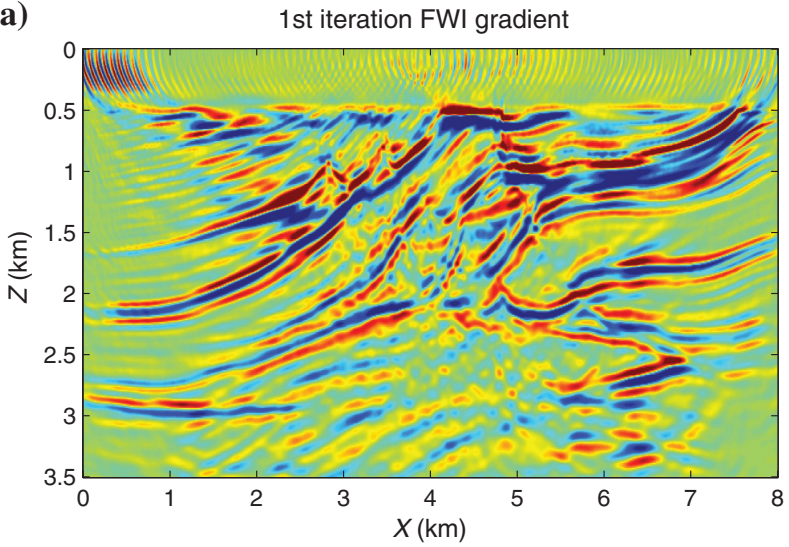

b)

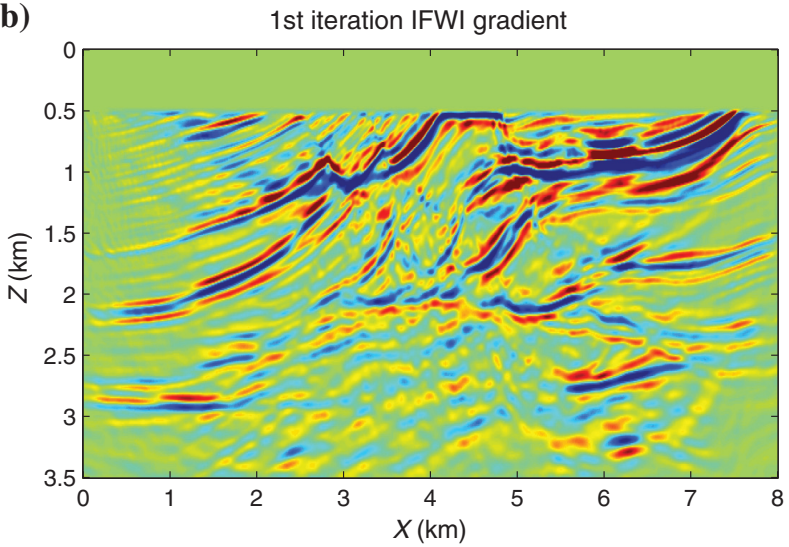

Figure 29. First iteration gradient of (a) FWI and (b) IFWI.
Figure 28. Well-log comparison at $X=2$ and $6 \mathrm{~km}$ for (a and c) FWI, (b and d) IFWI (shallow), and (e and f) IFWI (deep) tomograms. a)

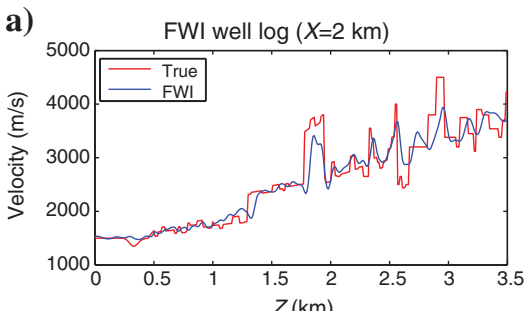

b)

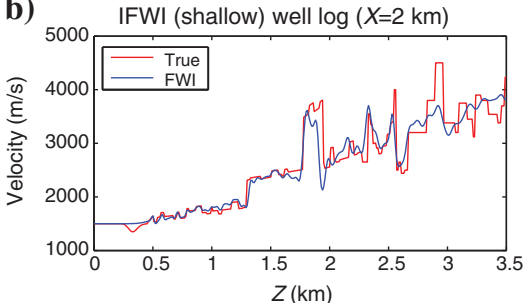

c)

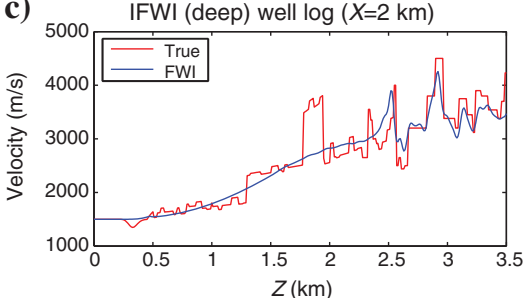

d)

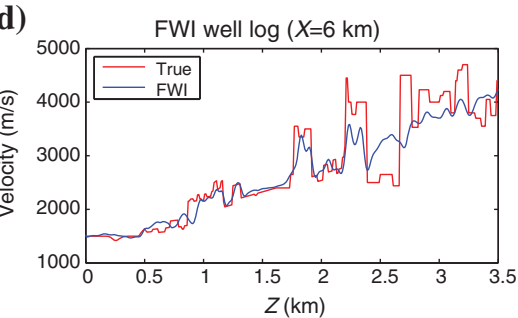

e)

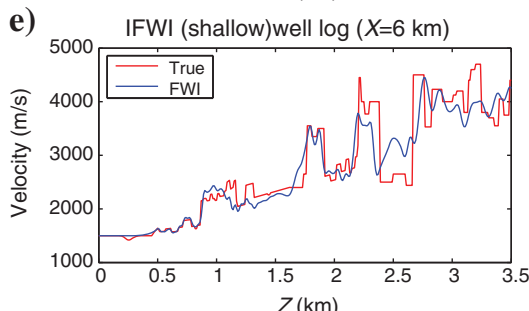

f)

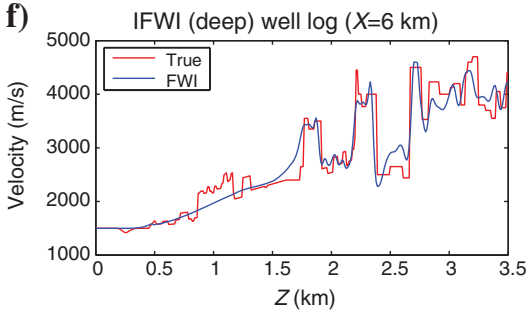


However, the seafloor topography can vary as fluids are extracted from the deeper reservoir. For example, 40 years of oil production from the Ekofisk field in Norway caused the seafloor to sink by $9 \mathrm{~m}$. Oil companies have thus deployed permanent acoustic sensors and geodetic instruments to monitor the subsidence and lateral movements (Ruiz et al., 2016). Hence, the availability of these measurements can provide a reliable estimate of the seabed as a reference reflector for the baseline and monitor surveys. Moreover, if discrepancies between the baseline and monitor migration images are extant in areas where no changes should have taken place, it is likely that these discrepancies have been caused by the overburden variations. These discrepancies in migration images could be investigated for choosing an appropriate reference interface, where the correct shape and location of the reference interface are estimated from the baseline migration image.

\section{CONCLUSION}

IFWI can largely overcome the challenges posed by an uncertain overburden velocity model and can mitigate cycle-skipping problems; it can also provide an accurate velocity model below a chosen reference reflector. FWI estimates the entire velocity model that explains all of the recorded data, which can lead to inconsistent updates. IM is used to evaluate the gradient at each iteration for IFWI, which mitigates the effect of errors in the overburden velocity and provides more reliable velocity updates in regions lying below the reference interface. Results with synthetic and field-data show that IFWI can overcome some challenges posed by an uncertain velocity model in the overburden and provide a more accurate velocity model below the overburden.

Potentially important applications of IFWI are target-oriented waveform and 4D inversion. Synthetic results suggest that IFWI can overcome the challenges posed by time-varying changes in the overburden by providing an accurate velocity model below the selected reference reflector. Owing to the nonlinearity of FWI/IFWI, a straightforward subtraction of baseline and monitor models can lead to false features in the estimated time-lapse changes in the reservoir. The double-difference approach provides a more accurate estimate of the velocity variations in the reservoir by exclusively inverting for time-lapse changes in the waveforms. A combination of double-difference and IFWI can provide a much more reliable estimate of the velocity change inside the reservoir.

The main challenge associated with IFWI is accurate identification of a suitable reference interface; errors in its estimation will translate into an erroneous tomogram. Large discrepancies in the depth of the reference reflector will lead to large errors in the kinematic corrections for the deeper reflectors, which can result in the predicted crosscorrelograms being cycle skipped with respect to the observed ones. This problem can be overcome by using a layerstripping approach, wherein deeper reflectors are selected as reference reflectors for inverting the deeper areas of the subsurface.

\section{ACKNOWLEDGMENTS}

The research reported in this publication was supported by the King Abdullah University of Science and Technology (KAUST) in Thuwal, Saudi Arabia. We would also like to thank the KAUST Supercomputing Laboratory (KSL) for providing access to supercomputing facilities. We are grateful to the sponsors of the Center for Subsurface Imaging and Modeling (CSIM) Consortium for their financial support.

\section{DATA AND MATERIALS AVAILABILITY}

Data associated with this research are confidential and cannot be released.

\section{APPENDIX A}

\section{SENSITIVITY ANALYSIS OF IFWI}

This section discusses the mitigation of cycle skipping by IFWI and its sensitivity with respect to the (1) source-receiver offset, (2) relative depth of target events, and (3) depth of the chosen reference reflector. Cycle skipping occurs when the arrival times of the observed and predicted waveforms differ by more than a half-cycle and causes FWI to get stuck in a local minimum. If the reflection traveltimes for the predicted and observed data for a target reflection point $\mathbf{x}_{0}$ are represented by $\tau_{\mathrm{obs}}^{x_{0}}$ and $\tau_{\mathrm{cal}}^{x_{0}}$, and the unresolved anomalies in the overburden are large enough to cause cycle skipping, then

$$
\left|\tau_{\mathrm{obs}}^{x_{0}}-\tau_{\mathrm{cal}}^{x_{0}}\right|>\frac{T}{2}
$$

where $T$ is the dominant period.

For the crosscorrelograms, the traveltimes for the observed and predicted waveforms are $\tau_{\text {obs }}^{x_{0}}-\tau_{\text {obs }}^{x_{\text {ref }}}$ and $\tau_{\text {cal }}^{x_{0}}-\tau_{\text {cal }}^{x_{\text {ref }}}$, respectively. The cycle-skipping criterion for the crosscorrelograms is modified to be

$$
|\tau_{\text {obs }}^{x_{0}}-\tau_{\text {cal }}^{x_{0}}+\overbrace{\tau_{\text {cal }}^{x_{\text {ref }}}-\tau_{\text {obs }}^{x_{\text {ref }}}}^{\delta_{\text {ref }}^{\text {timing error }}}|>\frac{T}{2},
$$

where $\delta^{\text {ref }}$ counters the errors induced in $\tau_{\text {obs }}^{x_{0}}-\tau_{\text {cal }}^{x_{0}}$ by fast- and slow-velocity anomalies and thus reduces the discrepancy between the predicted and observed crosscorrelograms. Therefore, the time differences between the observed and predicted crosscorrelated waveforms are reduced, so that cycle skipping is mitigated and there is less of a tendency to getting stuck in a local minimum.

The fidelity of the approximate redatuming operation deteriorates with an increase in the source-receiver offset and relative depth of the target event with respect to the reference interface. If the data honor the cycle-skipping criterion in equation A-2, IFWI will fall into a local minimum. Figure $1 \mathrm{a}$ and $1 \mathrm{~b}$ shows the model settings for the observed and predicted data, where the thickness and the velocity of the first layer are denoted as $d$ and $V$, respectively. In Figure 1a, the rays pass through very small elliptical anomalies, which causes distortions in the observed data. This assumes that the anomalies cause a deviation in the angles of incidence for the two reflection events at the first interface, but the effective background velocity for the first layer is $V$. The angles of incidence for the reference and target events are modified from $\theta_{1}$ and $\theta_{2}$ to $\hat{\theta}_{1}$ and $\theta_{2}$, respectively, so that the interferometric correction term $\delta^{\text {ref }}$ is given by

$$
\delta^{\mathrm{ref}}=\tau_{\text {cal }}^{x_{\text {ref }}}-\tau_{\text {obs }}^{x_{\text {ref }}}, \quad=\frac{2 d}{V}\left(\frac{1}{\cos \theta_{1}}-\frac{1}{\cos \theta_{1}^{\prime}}\right) .
$$

A very small velocity change in the shallow layer will result in very small fluctuations in $\theta_{1}$ and $\theta_{2}$ so that $\theta_{1}=\theta_{1}+\Delta \theta$ 
and ${ }^{\prime} \theta_{2}=\theta_{2}+\Delta \theta$. Substituting the perturbed value of $\dot{\theta}_{1}$ in equation $\mathrm{A}-3$, we get

$$
\begin{gathered}
\delta^{\mathrm{ref}}=\frac{2 d}{V}\left(\frac{1}{\cos \theta_{1}}-\frac{1}{\cos \left(\theta_{1}+\Delta \theta\right)}\right), \\
=\frac{2 d}{V}\left(\frac{1}{\cos \theta_{1}}-\frac{1}{\cos \theta_{1} \cos \Delta \theta-\sin \theta_{1} \sin \Delta \theta}\right),
\end{gathered}
$$

where $\Delta \theta$ is a measure of the velocity errors in the model used for generating the observed data. Using the small-angle approximations $\cos \Delta \theta=1$ and $\sin \Delta \theta=\Delta \theta$, equation A-4 simplifies to

$$
\begin{aligned}
\delta^{\mathrm{ref}} & =\frac{2 d}{V}\left[\frac{1}{\cos \theta_{1}}-\frac{1}{\cos \theta-\sin \theta_{1} \Delta \theta}\right], \\
& =\frac{2 d}{V \cos \theta_{1}}\left[1-\frac{1}{1-\tan \theta_{1} \Delta \theta}\right] .
\end{aligned}
$$

If $\Delta \theta$ and $\tan \theta_{1}$ are small enough so $\left|\tan \theta_{1} \Delta \theta\right|<1$, then $\frac{1}{1-\tan \theta_{1} \Delta \theta}$ can be expanded as an infinite geometric series. Ignoring higher order terms in $\Delta \theta$ could be written as

$$
\begin{aligned}
\delta^{\mathrm{ref}} & =\frac{2 d}{V \cos \theta_{1}}\left[1-\left(1+\tan \theta_{1} \Delta \theta\right)\right], \\
& =-\frac{2 d}{V \cos \theta_{1}}\left(\tan \theta_{1} \Delta \theta\right) .
\end{aligned}
$$

Similarly, the reflection-timing error $\delta t^{x_{0}}$ associated with the target point $x_{0}$ can be written as

$$
\delta t^{x_{0}}=-\frac{2 d}{V \cos \theta_{2}}\left(\tan \theta_{2} \Delta \theta\right) .
$$

This distortion is corrected by using the interferometric correction $\delta^{\text {ref }}$, so the discrepancy between the two is given by

$$
\text { error }=\delta t^{x_{0}}-\delta^{\mathrm{ref}}, \quad=-\frac{2 d}{V}\left[\left(\frac{\sin \theta_{2}}{\cos ^{2} \theta_{2}}-\frac{\sin \theta_{1}}{\cos ^{2} \theta_{1}}\right) \Delta \theta\right] .
$$

For small offsets and target reflectors in close vicinity of the reference reflector, $\theta_{2}$ and $\theta_{1}$ are almost equal to each other and the error term is small and IFWI will be effective for such reflectors. Increments in offset and depth of the target reflectors relative to the reference interface will result in an increase of $\theta_{2}$ and $\theta_{1}$, leading to a growth in the error term, which undermines the accuracy of the redatuming.

Another important factor that can influence the performance of IFWI is the depth of the chosen reference reflector. From equation $\mathrm{A}-8$, we can see that $\theta_{2}$ and $\theta_{1}$ are the two main components that govern the behavior of the error term. For a fixed maximum offset, deeper reference reflectors will possess lower values of $\theta_{1}$ and the deeper target-reflector's angle-of-incidence $\theta_{2}$ will also be small and closer to $\theta_{1}$. This implies that deeper reflectors would serve as a better choice for a reference reflector.

\section{REFERENCES}

Asnaashari, A., R. Brassier, S. Garambois, F. Audebert, P. Thore, and J. Virieux, 2012, Time-lapse imaging using regularized FWI: A robustness study: 82nd Annual International Meeting, SEG, Expanded Abstracts, doi: 10.1190/segam2012-0699.1.

Boonyasiriwat, C., G. T. Schuster, P. Valasek, and W. Cao, 2010, Applications of multiscale waveform inversion to marine data using a flooding technique and dynamic early-arrival windows: Geophysics, 75, no. 6, R129-R136, doi: 10.1190/1.3507237.

Bunks, C., F. Saleck, S. Zaleski, and G. Chavent, 1995, Multiscale seismic waveform inversion: Geophysics, 60, 1457-1473, doi: 10.1190/1 .1443880 .

Fichtner, A., J. Trampert, P. Cupillard, E. Saygin, T. Taymaz, Y. Capdeville, and A. Villaseñor, 2013, Multiscale full waveform inversion: Geophysical Journal International, 194, 534-556, doi: 10.1093/gji/ggt118.

Fu, L., B. Guo, and G. T. Schuster, 2018, Multiscale phase inversion of seismic data: Geophysics, 83, no. 2, R159-R171, doi: 10.1190/ geo2017-0353.1.

Guo, B., and G. Schuster, 2017, Wave-equation migration velocity analysis using plane-wave common-image gathers: Geophysics, 82, no. 5, S327S340, doi: $10.1190 / \mathrm{geo} 2016-0653.1$.

Huang, Y., 2013, Least-squares migration and full waveform inversion with multisource frequency selection: Ph.D. thesis, King Abdullah University of Science and Technology.

Huang, Y., and G. T. Schuster, 2014, Resolution limits for wave equation imaging: Journal of Applied Geophysics, 107, 137-148, doi: 10.1016/j .jappgeo.2014.05.018.

Jones, I. F., 2012, Tutorial: Incorporating near-surface velocity anomalies in pre-stack depth migration models: First Break, 30, 47-58, doi: 10.3997/ 1365-2397.2011041.

Kamei, R., and D. Lumley, 2017, Full waveform inversion of repeating seismic events to estimate time-lapse velocity changes: Geophysical Journal International, 209, 1239-1264, doi: 10.1093/gji/ggx057.

Lambaré, G., 2008, Stereotomography: Geophysics, 73, no. 5, VE25-VE34, doi: $10.1190 / 1.2952039$.

MacKay, S., J. Pried, and C. Carvill, 2003, The impact of water-velocity variations on deep-water seismic data: The Leading Edge, 22, 344350, doi: $10.1190 / 1.1572088$.

Maharramov, M., B. L. Biondi, and M. A. Meadows, 2016, Time-lapse inverse theory with applications: Geophysics, 81, no. 6, R485-R501, doi: 10.1190/geo2016-0131.1.

Murphy, G. E., and S. Gray, 1999, Manual seismic reflection tomography: Geophysics, 64, 1546-1552, doi: 10.1190/1.1444658.

Nocedal, J., and S. Wright, 2006, Numerical optimization: Springer.

Queisser, M., and S. C. Singh, 2013, Pull waveform inversion in the time lapse mode applied to $\mathrm{CO}_{2}$ storage at Sleipner: Geophysical Prospecting, 61, 537-555, doi: 10.1111/gpr.2013.61.issue-3.

Raknes, E. B., and B. Arntsen, 2014, Time-lapse full-waveform inversion of limited-offset seismic data using a local migration regularization: Geophysics, 79, no. 3, WA117-WA128, doi: 10.1190/geo2013-0369.1.

Routh, P., G. Palacharla, I. Chikichev, and S. Lazaratos, 2012, Full wavefield inversion of time-lapse data for improved imaging and reservoir characterization: 82nd Annual International Meeting, SEG, Expanded Abstracts, doi: 10.1190/segam2012-1043.1

Ruiz, H., R. Agersborg, L. Hille, M. Lien, and M. Vatshelle, 2016, Monitoring offshore reservoirs using 4D gravity and subsidence with improved tide corrections: 86th Annual International Meeting, SEG, Expanded Abstracts, 2946-2950, doi: 10.1190/segam2016-13576781.1.

Schleicher, J., J. C. Costa, and A. Novais, 2008, A comparison of imaging conditions for wave-equation shot-profile migration: Geophysics, 73, no. 6, S219-S227, doi: 10.1190/1.2976776.

Schuster, G., 2017, Seismic inversion: SEG.

Sheng, J., A. Leeds, M. Buddensiek, and G. Schuster, 2006, Early arrival waveform tomography on near-surface refraction data: Geophysics, 71, no. 4, U47-U57, doi: 10.1190/1.2210969.

Sinha, M., and G. Schuster, 2016, Mitigation of defocusing by statics and near-surface velocity errors by interferometric least-squares migration with a reference datum: Geophysics, 81, no. 4, S195-S206, doi: 10 $.1190 /$ geo2015-0637.1.

Watanabe, T., S. Shimizu, E. Asakawa, and T. Matsuoka, 2005, Differential waveform tomography for timelapse crosswell seismic data with application to gas hydrate production monitoring: 75 th Annual International Meeting, SEG, Expanded Abstracts, 2323-2326.

Yang, D., F. Liu, S. Morton, A. Malcolm, and M. Fehler, 2016, Time-lapse full-waveform inversion with ocean-bottom-cable data: Application on Valhall field: Geophysics, 81, no. 4, R225-R235, doi: 10.1190/ geo2015-0345.1.

Zhou, M., Z. Jiang, J. Yu, and G. Schuster, 2006, Comparison between interferometric migration and reduced-time migration of commondepth-point data: Geophysics, 71, no. 4, SI189-SI196, doi: 10.1190/1 .2213046 . 